\title{
Stereoselective synthesis and structural analysis of polycyclic lactams derived from tetrahydroisoquinoline 1,2- and 1,3-diamines
}

\author{
Henri Kivelä,,${ }^{a} *$ Olli Martiskainen, ${ }^{a}$ Kalevi Pihlaja, ${ }^{a}$ Zita Zalán, ${ }^{b}$ and László Lázár ${ }^{b, *}$ \\ ${ }^{a}$ Department of Chemistry, Structural Chemistry Group, University of Turku, \\ FI-20014, Finland \\ ${ }^{b}$ Institute of Pharmaceutical Chemistry, University of Szeged, Eötvös u. 6., \\ H-6720 Szeged, Hungary \\ E-mail: hemiki@utu.fi, lazar@pharm.u-szeged.hu
}

Dedicated to Professor Ferenc Fülöp on the occasion of his 60th birthday

\begin{abstract}
By means of the domino ring-closure reactions of $\left(1 S^{*}, 1^{\prime} S^{*}\right)-1-\left(1^{\prime}\right.$-aminoethyl)-, $\left(1 R^{*}, 1^{\prime} R^{*}\right)-1$ (2'-amino-1'-methylethyl)- and 1-(2-aminophenyl)-6,7-dimethoxy-1,2,3,4-tetrahydroisoquinoline with acyclic and aromatic $\gamma$ - or $\delta$-oxo acids, angularly-condensed tetra-, penta- and hexacyclic lactam derivatives were formed with practically full diastereoselectivities (de $\sim 100 \%$ ), containing the substituents at the annelations of the saturated heterocyclic rings in cis position.

The structure and relative stereochemistry of the products were determined with ${ }^{1} \mathrm{H}$ and ${ }^{13} \mathrm{C}$ NMR spectroscopy. Further, the tetrahydropyridine ring (ring B) was observed to prefer cis fusion with the condensed imidazolidine or hexahydropyrimidine ring (ring $\mathrm{C}$ ), with one exception preferring trans fusion (namely, the tetracyclic imidazolidine derivative bearing a methyl substituent at the $\mathrm{C} / \mathrm{D}$ ring annelation, cmpd. 11). These two conformations can interconvert via simultaneous nitrogen inversion at the $\mathrm{B} / \mathrm{C}$ annelation and ring inversion of the ring $\mathrm{B}$, and their populations could be roughly estimated from the $J$-coupling constant data for each lactam derivative. The compounds gave fragment ions that were typical for the structures of the compounds.
\end{abstract}

Keywords: Diamine, isoquinoline, lactam, domino reaction, ${ }^{1} \mathrm{H}$ and ${ }^{13} \mathrm{C} \mathrm{NMR}$, electron impact mass spectrometry

\section{Introduction}

The cyclocondensation of various 1,2-difunctional compounds (1, amino alcohols, amino thiols or diamines, containing a primary amino group) comprise a well-established method for the 
preparation of 1,3-heterocycle-fused $\gamma$ - or $\delta$-lactams with a nitrogen at the annelation (4). ${ }^{1}$ The reaction is classified as a domino process, ${ }^{2}$ since it presumably occurs in two steps: first a ringchain tautomeric intermediate $\mathbf{3 A - 3 B}$ ) is formed, the equilibrium of which gradually shifts towards the cyclic form $\mathbf{4}$ in consequence of the practically irreversible intramolecular $N$ acylation. ${ }^{3}$ The nitrogen-bridged $\gamma$ - or $\delta$-lactams $\mathbf{4}$, both in racemic and in enantiomerically pure form, are often applied as intermediates in numerous synthetic processes involving various regioand stereocontrolled transformations. ${ }^{4}$

We recently described the domino ring-closures of 1-(aminomethyl)- and 1-(2-aminoethyl)6,7-dimethoxy-1,2,3,4-tetrahydroisoquinoline with $\gamma$ - and $\delta$-oxo acids, whereby tetra- and pentacyclic either angularly or linearly tetrahydroisoquinoline-condensed lactam derivatives were formed with excellent diastereoselectivities. ${ }^{5}$ As a continuation of this work and in connection with our previous studies on the synthesis and structural analysis of tetrahydroisoquinolinecondensed saturated heterocycles, ${ }^{6}$ we now report on the reactions of 1-substituted 1,2,3,4tetrahydroisoquinoline 1,2- and 1,3-diamines, bearing a methyl substituent or an aromatic ring in the side chain, with $\gamma$ - and $\delta$-oxo acids. Our aim was to investigate the effects of the side chain substituents and the size of the lactam rings formed on the stereochemical outcome of the reactions, and the conformational and mass spectral behaviour of the tetra- and pentacyclic products.

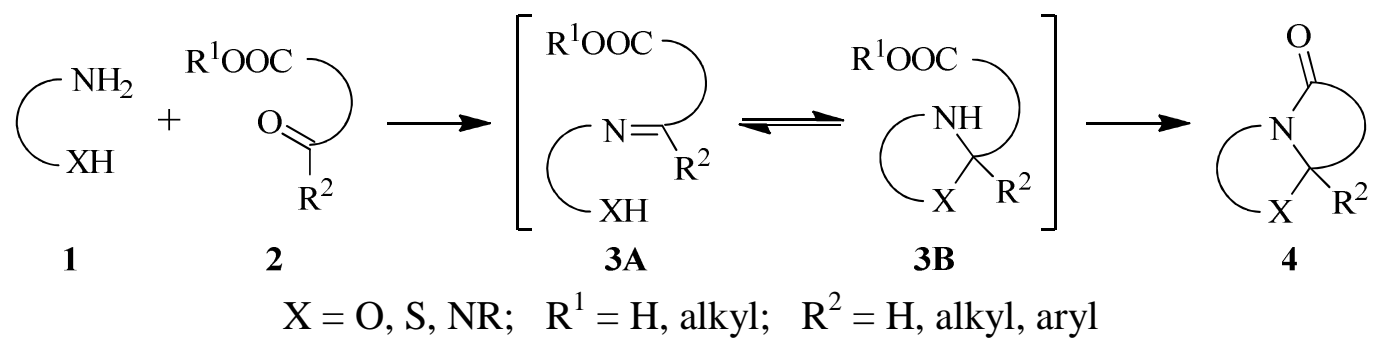

\section{Scheme 1}

\section{Results and Discussion}

\section{Synthesis}

From the starting diamines for the preparation of the target polycyclic lactams, $\left(1 R^{*}, 1^{\prime} R^{*}\right)-1-\left(2^{\prime}-\right.$ amino-1'-methylethyl)-6,7-dimethoxy-1,2,3,4-tetrahydroisoquinoline ${ }^{7}$ (10) and 1-(2-aminophenyl)-6,7-dimethoxy-1,2,3,4-tetrahydroisoquinoline ${ }^{8}$ (21) were synthesized according to known procedures. (1 $\left.S^{*}, 1^{\prime} S^{*}\right)-1$-(1'-Aminoethyl)-6,7-dimethoxy-1,2,3,4-tetrahydroisoquinoline (9) was prepared in five steps, similarly to its homologue 10, starting from $N$-Cbz-DL-alanine (5) and homoveratrylamine (Scheme 2). Reduction of 7 with $\mathrm{NaBH}_{4}$ gave a ca. 8:1 mixture of tetrahydroisoquinoline diastereomers $8 \mathbf{a}$ and $\mathbf{8 b}$, from which $\mathbf{8 a}$ could be obtained by crystallization and was converted to the $1 S^{*}, 1^{\prime} S^{*}$ diamine diastereomer 9. Diastereomeric ratios 
8a:8b were determined from the ${ }^{1} \mathrm{H}$ NMR spectra by integration of the well separated 1 '-Me doublets. The relative configuration of 9 was deduced based on its ring-closed lactam derivatives (see below). ${ }^{9}$

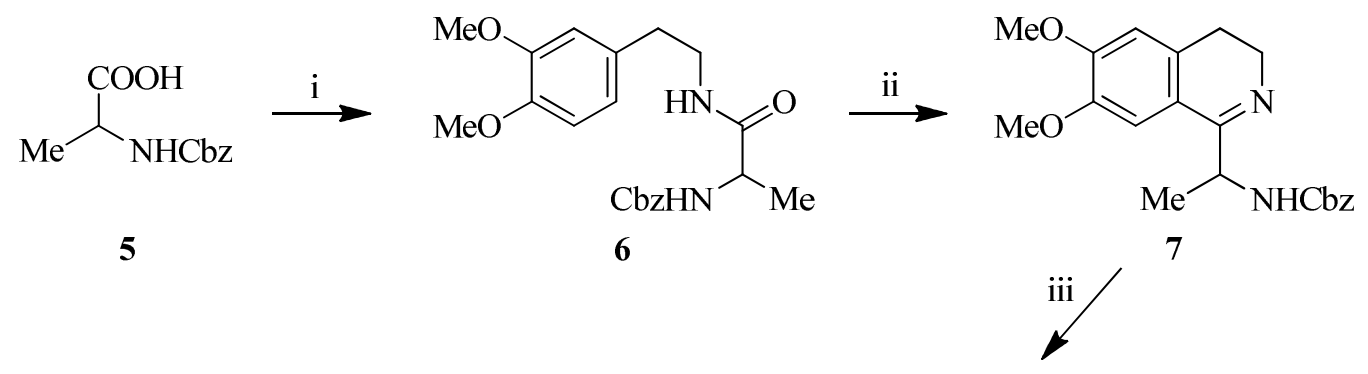

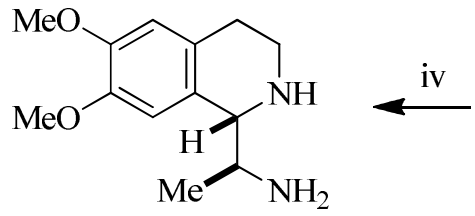

9

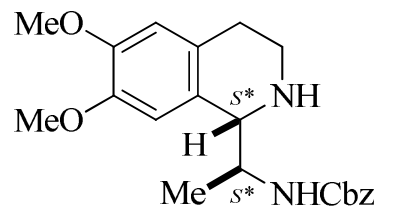

8a

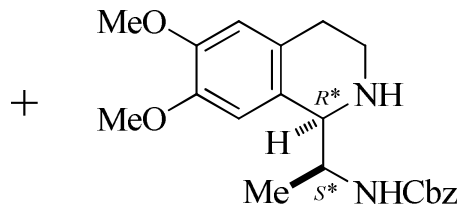

$\mathbf{8 b}$

Scheme 2. Reagents and conditions: (i) 1: $\mathrm{Et}_{3} \mathrm{~N}, \mathrm{ClCOOEt}$, toluene, $-10{ }^{\circ} \mathrm{C}, 5 \mathrm{~min}, 2$. homoveratrylamine, $\mathrm{CH}_{2} \mathrm{Cl}_{2}, 0^{\circ} \mathrm{C}$ then reflux, $5 \mathrm{~min}, 79 \%$; (ii): $\mathrm{POCl}_{3}, \mathrm{CHCl}_{3}$, reflux, $3 \mathrm{~h}, 75 \%$; (iii): $\mathrm{NaBH}_{4}, \mathrm{MeOH}, 0{ }^{\circ} \mathrm{C}, 3$ h, then r.t., 3 h, 8a:8b = 8:1, 8a: 74\%; (iv): $33 \% \mathrm{HBr}$ in AcOH, r.t., 30 min, then $\mathrm{NaOH}, 83 \%$.

When tetrahydroisoquinoline diamines 9 and $\mathbf{1 0}$ were boiled in toluene with 4-oxopentanoic acid, 3-benzoylpropanoic acid, 4-benzoylbutanoic acid, 2-formylbenzoic acid or 2-benzoylbenzoic acid, cyclocondensations generally took place within 2-12 $\mathrm{h}$ to furnish the corresponding angularly-condensed tetra- and pentacyclic lactams 11-20 (Scheme 3) in good yields. By a similar reaction of diamine $\mathbf{2 1}$ and 2-formylbenzoic acid, the angularly-condensed hexacyclic lactam 22 was obtained (Scheme 4). NMR spectra of the crude products 11-20 and 22 indicated that neither the methyl substituent nor the aromatic ring at the side chain influenced significantly the stereochemical outcome of the lactam formation, since the polycycles 11-20 and 22 were formed with practically full diastereoselectivity ( $d e \sim 100 \%$ ), affording only the isomers having the hydrogen at the annelation of the tetrahydroisoquinoline and imidazolidine/hexahydropyrimidine rings (H-10b for 11-14, $\mathrm{H}-11 \mathrm{~b}$ for 15 and 16, H-4b for 17-20 and 22) and substituent $\mathrm{R}$ ( $\mathrm{H}$ at $14 \mathrm{~b}$ in 22) in the cis position. The observed high de values in favour of the formation of the cis lactams can be explained as a result of the kinetic control governing the second cyclization steps of the domino cyclocondensations. ${ }^{3,5}$ 


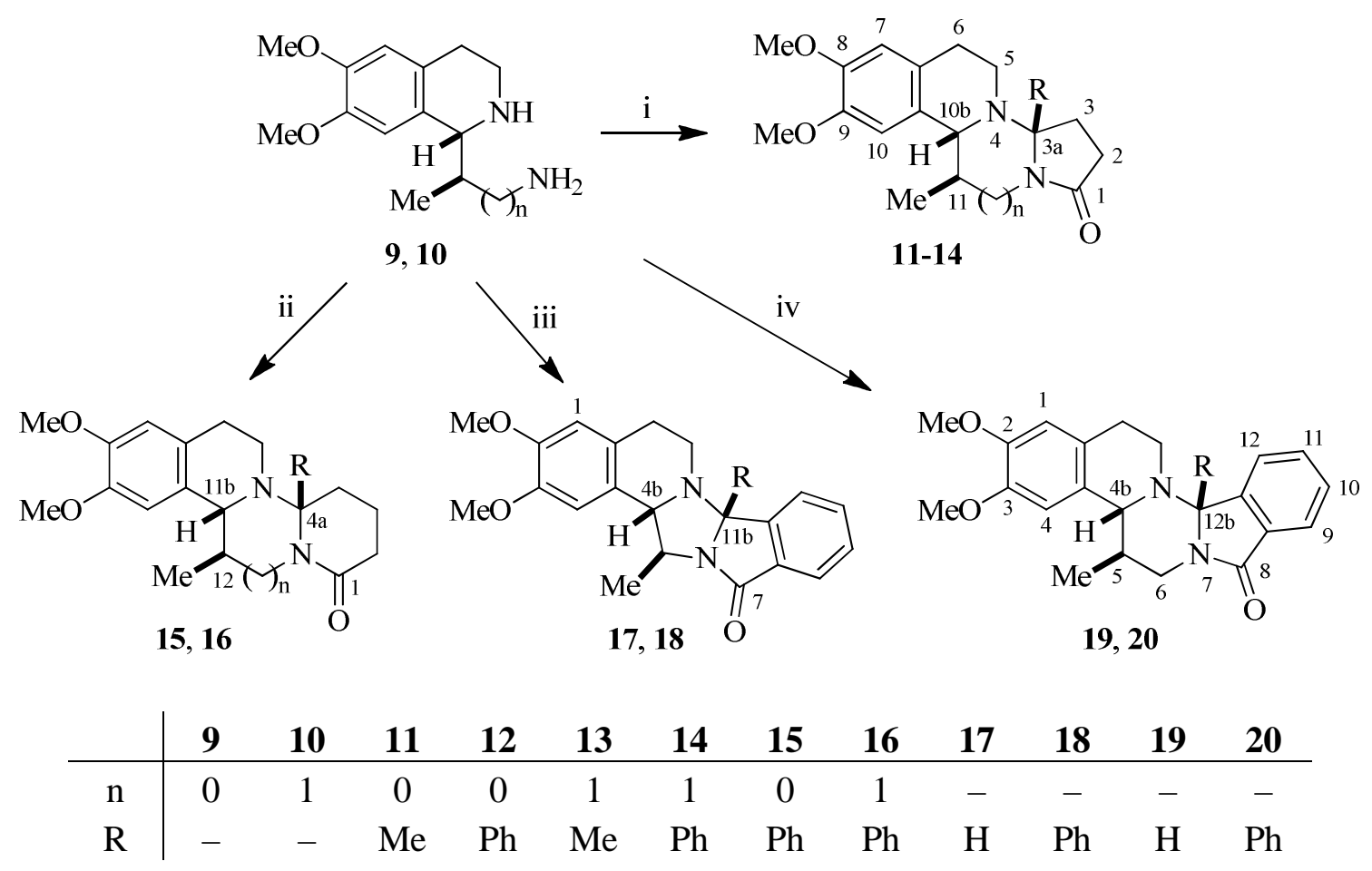

Scheme 3. Reagents and conditions: (i) $\mathrm{RCO}\left(\mathrm{CH}_{2}\right)_{2} \mathrm{COOH}$, toluene, reflux, 4-8 h, 59-77\%; (ii) $\mathrm{PhCO}\left(\mathrm{CH}_{2}\right)_{3} \mathrm{COOH}$, toluene, reflux, 10-12 h, 52-63\%; (iii) 2-RCO- $\mathrm{C}_{6} \mathrm{H}_{4} \mathrm{COOH}$, toluene, reflux, 2-3 h, 63-74\%; (iv) 2-RCO- $\mathrm{C}_{6} \mathrm{H}_{4} \mathrm{COOH}$, toluene, reflux, $2-3 \mathrm{~h}, 58-76 \%$.

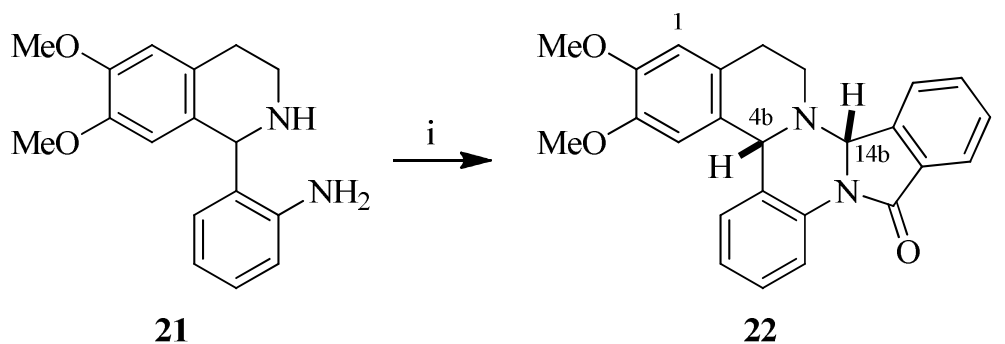

Scheme 4. Reagents and conditions: (i) 2-HCO- $\mathrm{C}_{6} \mathrm{H}_{4} \mathrm{COOH}$, toluene, PTSA, reflux, $12 \mathrm{~h}, 62 \%$.

\section{NMR spectroscopic structure determination}

The solution structures of products 11-20 and 22 were determined by ${ }^{1} \mathrm{H}$ and ${ }^{13} \mathrm{C}$ NMR spectroscopy in $\mathrm{CDCl}_{3}$ solution. The ${ }^{1} \mathrm{H}$ and ${ }^{13} \mathrm{C}$ chemical shifts of these compounds were assigned with the help of standard 2D NMR correlation spectra (dqf-COSY, NOESY, multiplicity-edited HSQC and HMBC). The stereo assignment of diastereotopic protons, and the analysis of relative configuration and conformation was based on NOEs and $J_{\mathrm{H}, \mathrm{H}}$-coupling constant values. Accurate ${ }^{1} \mathrm{H}$ chemical shifts and $J_{\mathrm{H}, \mathrm{H}}$-coupling constants were obtained from computer simulation and iteration of the $1 \mathrm{D}{ }^{1} \mathrm{H}$ NMR spectra. The NMR data are presented in 
Tables 1-3. Below, the diastereotopic hydrogens which are on the same side of the polycycle as the isoquinoline bridgehead hydrogen will be labelled as syn or $s$, and as anti or $a$ otherwise.

Compounds 11-14 contain three asymmetric carbons (C3a, C10b, C11), as do 15-16 (C4a, C11b, C12), 17-18 (C4b, C5, C11b) and 19-20 (C4b, C5, C12b), whereas 22 contains two (C4b, $\mathrm{C} 14 \mathrm{~b}$ ). Of these, the R-bearing carbon atom ( $\mathrm{C} 3 \mathrm{a}$ for $\mathbf{1 1}$ etc.) is a new stereo centre formed during the domino ring-closure and its configuration is, a priori, unknown. The syntheses were highly stereo-selective, so that only one of the two possible epimers was produced to a notable extent. For each product, proton-proton NOE correlations proved that the relative positions of the substituents carried by the three asymmetric carbons (two for 22) is all-cis, as shown in Schemes 2 and 3. These NOEs also verify the configuration of the starting diamines $\mathbf{9}$ and $\mathbf{1 0 .}$

Table 1. Proton chemical shifts $\delta_{\mathrm{H}}$ (in ppm, $\mathrm{CDCl}_{3}, 298 \mathrm{~K}$ ) of 11-22. The label $s(a)$ indicates that the hydrogen is syn (anti) with respect to the isoquinoline bridgehead hydrogen. The chemical shifts marked with an asterisk (*) may be interchanged

\begin{tabular}{|c|c|c|c|c|c|c|c|}
\hline & 11 & 12 & 13 & 14 & & 15 & 16 \\
\hline $\mathrm{R}$ & $\mathrm{Me}$ & $\mathrm{Ph}$ & $\mathrm{Me}$ & $\mathrm{Ph}$ & $\mathrm{R}$ & $\mathrm{Ph}$ & $\mathrm{Ph}$ \\
\hline $\mathrm{n}$ & 0 & 0 & 1 & 1 & $\mathrm{n}$ & 0 & 1 \\
\hline $2 s$ & 2.81 & 2.82 & 2.49 & 2.52 & $2 s$ & 2.57 & 2.50 \\
\hline \multirow[t]{3}{*}{$2 a$} & 2.45 & 2.51 & 2.50 & 2.58 & $2 a$ & 2.39 & 2.52 \\
\hline & & & & & $3 s$ & 1.52 & 1.49 \\
\hline & & & & & $3 a$ & 1.70 & 1.68 \\
\hline $3 s$ & 2.02 & 2.05 & 2.08 & 1.98 & $4 s$ & 1.99 & 1.77 \\
\hline $3 a$ & 2.17 & 2.73 & 2.33 & 2.49 & $4 a$ & 2.15 & 2.51 \\
\hline $5 s$ & 2.69 & 3.18 & 2.88 & 3.20 & $6 s$ & 3.32 & 3.59 \\
\hline $5 a$ & 2.95 & 2.50 & 2.95 & 3.13 & $6 a$ & 2.65 & 3.22 \\
\hline $6 s$ & 2.82 & 3.09 & 2.90 & 3.15 & $7 s$ & 3.09 & 3.12 \\
\hline $6 a$ & 3.01 & 2.81 & 2.82 & 2.85 & $7 a$ & 2.80 & 2.88 \\
\hline 7 & $6.63^{*}$ & 6.63 & 6.60 & 6.65 & 8 & 6.62 & 6.65 \\
\hline 8-OMe & 3.84 & 3.84 & 3.84 & 3.86 & 9-OMe & 3.84 & 3.86 \\
\hline 9-OMe & 3.84 & 3.76 & 3.84 & 3.75 & 10-OMe & 3.75 & 3.75 \\
\hline 10 & $6.62 *$ & 6.44 & 6.59 & 6.31 & 11 & 6.46 & 6.30 \\
\hline $10 \mathrm{~b}$ & 3.86 & 3.77 & 4.06 & 3.62 & $11 b$ & 3.50 & 3.48 \\
\hline $11 a$ & 3.91 & 3.80 & 2.01 & 2.12 & $12 a$ & 3.95 & 2.16 \\
\hline 11-Me & 1.61 & 1.46 & 1.02 & 0.78 & $12-\mathrm{Me}$ & 1.54 & 0.77 \\
\hline $12 s$ & - & - & 2.76 & 2.70 & $13 s$ & - & 2.55 \\
\hline $12 a$ & - & - & 4.10 & 4.19 & $13 a$ & - & 4.66 \\
\hline \multirow[t]{4}{*}{$\mathrm{R}$} & 1.42 & $7.62 o$ & 1.67 & $7.49 o$ & $\mathrm{R}$ & $7.54 o$ & 7.1(br) $o$ \\
\hline & & $7.36 \mathrm{~m}$ & & $7.38 \mathrm{~m}$ & & $7.35 \mathrm{~m}$ & $7.6(\mathrm{br}) o^{\prime}$ \\
\hline & & $7.30 p$ & & $7.30 p$ & & $7.30 p$ & $7.36 \mathrm{~m}$ \\
\hline & & & & & & & $7.29 p$ \\
\hline
\end{tabular}


Table 1. (Continued)

\begin{tabular}{|c|c|c|c|c|c|c|c|}
\hline & 17 & 18 & & 19 & 20 & & 22 \\
\hline $\mathrm{R}$ & $\mathrm{H}$ & $\mathrm{Ph}$ & $\mathrm{R}$ & $\mathrm{H}$ & $\mathrm{Ph}$ & $\mathrm{R}$ & $\mathrm{H}$ \\
\hline $\mathrm{n}$ & 0 & 0 & $\mathrm{n}$ & 1 & 1 & $\mathrm{n}$ & - \\
\hline 1 & 6.60 & 6.62 & 1 & 6.60 & 6.62 & 1 & 6.65 \\
\hline 2-OMe & 3.84 & 3.84 & 2-OMe & 3.85 & 3.85 & 2-OMe & 3.86 \\
\hline 3-OMe & 3.88 & 3.79 & 3-OMe & 3.87 & 3.79 & 3-OMe & 3.98 \\
\hline 4 & 6.67 & 6.51 & 4 & 6.62 & 6.42 & 4 & 6.89 \\
\hline $4 b$ & 4.18 & 4.12 & $4 b$ & 3.85 & 3.79 & $4 b$ & 5.51 \\
\hline $5 a$ & 3.85 & 3.96 & $5 a$ & 2.19 & 2.20 & 5 & 7.28 \\
\hline \multirow[t]{3}{*}{$5-\mathrm{Me}$} & 1.69 & 1.55 & $5-\mathrm{Me}$ & 1.05 & 0.88 & 6 & 7.05 \\
\hline & & & $6 s$ & 2.96 & 2.85 & 7 & 7.33 \\
\hline & & & $6 a$ & 4.45 & 4.49 & 8 & 8.71 \\
\hline 8 & 7.83 & 7.81 & 9 & 7.87 & 7.87 & 11 & 7.96 \\
\hline 9 & 7.54 & 7.44 & 10 & 7.53 & 7.40 & 12 & 7.60 \\
\hline 10 & 7.61 & 7.46 & 11 & 7.58 & 7.43 & 13 & 7.67 \\
\hline 11 & 7.63 & 7.23 & 12 & 7.60 & 7.21 & 14 & 7.71 \\
\hline $13 s$ & 2.39 & 2.49 & $14 s$ & 2.38 & 2.54 & $16 s$ & 2.32 \\
\hline $13 a$ & 2.18 & 2.08 & $14 a$ & 2.78 & 2.88 & $16 a$ & 2.44 \\
\hline $14 s$ & 2.90 & 3.04 & $15 s$ & 2.89 & 3.00 & $17 s$ & 2.91 \\
\hline $14 a$ & 2.60 & 2.57 & $15 a$ & 2.63 & 2.64 & $17 a$ & 2.56 \\
\hline \multirow[t]{4}{*}{$\mathrm{R}$} & 5.65 & $7.80 o$ & $\mathrm{R}$ & 5.58 & 7.2(br)o & $\mathrm{R}$ & 6.08 \\
\hline & & $7.35 \mathrm{~m}$ & & & $8.1(\mathrm{br}) o^{\prime}$ & & \\
\hline & & $7.30 p$ & & & 7.36(br) $m$ & & \\
\hline & & & & & $7.29 p$ & & \\
\hline
\end{tabular}

The preferred conformations of the polycyclic products could be deduced from the $J$ coupling constants and NOE correlations between protons, and by using the structural information thus obtained as constraints in molecular mechanics modelling. Compound 11 showed an NOE correlation between all pairs of $3 \mathrm{a}-\mathrm{Me}, 5-\mathrm{H}_{\text {syn }}$ and $10 \mathrm{~b}-\mathrm{H}$, indicating their mutual spatial proximity, and also displayed large ${ }^{3} J_{\mathrm{H}, \mathrm{H}}(5 s, 6 a)$ and ${ }^{3} J_{\mathrm{H}, \mathrm{H}}(10 \mathrm{~b}, 11 a)$ coupling values implying these hydrogens to be antiperiplanar. On the other hand, the $5-\mathrm{H}_{\text {syn }}$ proton of 12 14, and the analogous proton of derivatives $15-20$ and $22\left(6-\mathrm{H}_{s y n}\right.$ of 15 etc.), typically did not share an NOE correlation with the substituent $\mathrm{R}$ or the isoquinoline bridgehead proton. Instead, these derivatives showed an NOE between 5- $\mathrm{H}_{\text {anti }}$ and 11- $\mathrm{H}_{\text {anti }}(\mathbf{1 2}-\mathbf{1 4}), 6-\mathrm{H}_{\text {anti }}$ and $12-\mathrm{H}_{\text {anti }}(\mathbf{1 5}-$ 16) etc. The derivatives 12-20 and 22 also had large ${ }^{3} J_{\mathrm{H}, \mathrm{H}}(5 a, 6 s)(\mathbf{1 2}-\mathbf{1 4}),{ }^{3} J_{\mathrm{H}, \mathrm{H}}(6 a, 7 s)(\mathbf{1 5}-\mathbf{1 6})$ etc. values (as opposed to large ${ }^{3} J_{\mathrm{H}, \mathrm{H}}(5 s, 6 a)$ etc.). These data suggest two conformations populated by these compounds: 11 prefers a conformation in which the ring $\mathrm{B}$ (tetrahydropyridine) is approximately trans-fused with the ring $\mathrm{C}$ (imidazolidine) whilst the other derivatives prefer a cis-fused conformation between rings $\mathrm{B}$ and $\mathrm{C}$ (the latter being either 
imidazolidine, $\mathrm{n}=0$, or hexahydropyrimidine, $\mathrm{n}=1$ ). Ring $\mathrm{B}$ adopts a half-chair conformation with the two aromatic carbons and the aliphatic carbons attached to them defining a plane, and the remaining methylene carbon (C5 etc.) and nitrogen (N4 etc.) lying above and below this plane. In 11, it is the methylene carbon (C5) which lies on the same side of the plane as the bridgehead hydrogen $(10 \mathrm{~b}-\mathrm{H})$, whereas in 12-20 and 22 the syn atom is nitrogen (12-14: N4, 15-16: N5, 17-18: N12, 19-20: N13, 22: N15). These trans-B/C and cis-B/C conformations are depicted in Figures 1 and 2 for $\mathbf{1 1}$ and 16, respectively.

There is a possibility for an equilibrium between the trans-B/C and cis-B/C conformers, involving the ring inversion of ring $\mathrm{B}$ combined with an umbrella inversion of its nitrogen atom. In a pure trans-B/C conformation, ${ }^{3} J_{\mathrm{H}, \mathrm{H}}(5 s, 6 a)(\mathbf{1 1 - 1 4})$ or the analogous coupling (15-20, 22) would be large while the corresponding ${ }^{3} J_{\mathrm{H}, \mathrm{H}}(5 a, 6 s)$ etc. coupling would be small. In the $c i s-\mathrm{B} / \mathrm{C}$ conformation, the opposite would be true. In case of a fast equilibrium, the observed values of these coupling constants become population-weighted averages of the "large" (ca. 12.2 Hz) and

Table 2. Proton-proton coupling constants $J_{\mathrm{H}, \mathrm{H}}\left(\mathrm{in} \mathrm{Hz}, \mathrm{CDCl}_{3}, 298 \mathrm{~K}\right.$ ) of 11-22. The label $s(a)$ indicates that the hydrogen is syn (anti) with respect to the bridgehead hydrogen

\begin{tabular}{crrrrrrr}
\hline & $\mathbf{1 1}$ & $\mathbf{1 2}$ & $\mathbf{1 3}$ & $\mathbf{1 4}$ & & $\mathbf{1 5}$ & $\mathbf{1 6}$ \\
\hline $\mathrm{R}$ & $\mathrm{Me}$ & $\mathrm{Ph}$ & $\mathrm{Me}$ & $\mathrm{Ph}$ & $\mathrm{R}$ & $\mathrm{Ph}$ & $\mathrm{Ph}$ \\
$\mathrm{n}$ & 0 & 0 & 1 & 1 & $\mathrm{n}$ & 0 & 1 \\
\hline $2 s, 2 a$ & -17.0 & -17.2 & -17.5 & -17.6 & $2 s, 2 a$ & -18.4 & -18.2 \\
$2 s, 3 s$ & 9.6 & 10.3 & 10.4 & 10.6 & $2 s, 3 s$ & 6.6 & 5.1 \\
$2 s, 3 a$ & 10.3 & 7.5 & 4.5 & 2.8 & $2 s, 3 a$ & 1.5 & 4.6 \\
$2 a, 3 s$ & 2.4 & 4.5 & 7.3 & 8.9 & $2 a, 3 s$ & 11.4 & 10.1 \\
$2 a, 3 a$ & 10.2 & 10.4 & 10.3 & 10.1 & $2 a, 3 a$ & 7.8 & 6.2 \\
$3 s, 3 a$ & -12.7 & -14.1 & -14.2 & -14.8 & $3 s, 3 a$ & -12.8 & -13.7 \\
& & & & & $3 s, 4 s$ & 3.0 & 2.9 \\
& & & & & $3 s, 4 a$ & 13.4 & 10.9 \\
& & & & & $3 a, 4 s$ & 4.0 & 6.6 \\
& & & & & $3 a, 4 a$ & 3.3 & 3.3 \\
$5 s, 5 a$ & -10.8 & -10.2 & -11.5 & -11.3 & $6 s, 6 a$ & -12.5 & -13.6 \\
$5 s, 6 s$ & 5.0 & 5.3 & 6.7 & 7.5 & $6 s, 7 s$ & 5.3 & -11.7 \\
$5 s, 6 a$ & 10.3 & 2.8 & 3.3 & 1.3 & $6 s, 7 a$ & 2.5 & 1.8 \\
$5 a, 6 s$ & 2.5 & 11.2 & 9.9 & 11.6 & $6 a, 7 s$ & 11.7 & 10.8 \\
$5 a, 6 a$ & 7.0 & 3.8 & 5.1 & 4.9 & $6 a, 7 a$ & 3.7 & 5.2 \\
$6 s, 6 a$ & -16.4 & -16.0 & -16.3 & -16.4 & $7 s, 7 a$ & -16.1 & -16.5 \\
$10 \mathrm{~b}, 11 a$ & 8.2 & 9.7 & 10.6 & 10.8 & $11 b, 12 a$ & 9.5 & 10.8 \\
$11 a, 11-\mathrm{Me}$ & 6.3 & 6.3 & 6.6 & 6.5 & $12 a, 12-\mathrm{Me}$ & 6.1 & 6.5 \\
$11 a, 12 s$ & - & - & 11.9 & 11.8 & $12 a, 13 s$ & - & 12.0 \\
$11 a, 12 a$ & - & - & 5.3 & 5.2 & $12 a, 13 a$ & - & 5.0 \\
$12 s, 12 a$ & - & - & -13.5 & -13.3 & $13 s, 13 a$ & - & -13.7 \\
\hline
\end{tabular}


Table 2. (Continued)

\begin{tabular}{crrrrrrr}
\hline & $\mathbf{1 7}$ & $\mathbf{1 8}$ & & $\mathbf{1 9}$ & $\mathbf{2 0}$ & & $\mathbf{2 2}$ \\
\hline $\mathrm{R}$ & $\mathrm{H}$ & $\mathrm{Ph}$ & $\mathrm{R}$ & $\mathrm{H}$ & $\mathrm{Ph}$ & $\mathrm{R}$ & $\mathrm{H}$ \\
$\mathrm{n}$ & 0 & 0 & $\mathrm{n}$ & 1 & 1 & $\mathrm{n}$ & - \\
\hline $4 \mathrm{~b}, 5 a$ & 8.6 & 8.7 & $4 \mathrm{~b}, 5 a$ & 10.3 & 10.7 & 5,6 & 7.8 \\
$5 a, 5-\mathrm{Me}$ & 6.2 & 6.3 & $5 a, 5-\mathrm{Me}$ & 6.6 & 6.6 & 5,7 & 1.3 \\
& & & $5 a, 6 s$ & 11.5 & 11.7 & 6,7 & 7.4 \\
& & & $5 a, 6 a$ & 5.3 & 5.2 & 6,8 & 1.3 \\
8,9 & & & $6 s, 6 a$ & -13.4 & -13.4 & 7,8 & 8.2 \\
8,10 & 7.6 & 7.6 & 9,10 & 7.6 & 7.6 & 11,12 & 7.6 \\
9,10 & 1.1 & 1.2 & 9,11 & 1.1 & 1.1 & 11,13 & 1.1 \\
9,11 & 7.5 & 7.4 & 10,11 & 7.5 & 7.4 & 12,13 & 7.4 \\
10,11 & 1.0 & 1.0 & 10,12 & 1.0 & 1.0 & 12,14 & 0.9 \\
$13 s, 13 a$ & 7.6 & 7.6 & 11,12 & 7.5 & 7.6 & 13,14 & 7.6 \\
$13 s, 14 s$ & -11.0 & -10.9 & $14 s, 14 a$ & -12.0 & -11.9 & $16 s, 16 a$ & -11.6 \\
$13 s, 14 a$ & 5.6 & 5.4 & $14 s, 15 s$ & 7.8 & 7.7 & $16 s, 17 s$ & 6.9 \\
$13 a, 14 s$ & 3.3 & 1.9 & $14 s, 15 a$ & 1.0 & 1.5 & $16 s, 17 a$ & 1.4 \\
$13 a, 14 a$ & 10.5 & 12.2 & $14 a, 15 s$ & 11.4 & 11.3 & $16 a, 17 s$ & 11.8 \\
$14 s, 14 a$ & 4.2 & 3.4 & $14 a, 15 a$ & 5.3 & 5.0 & $16 a, 17 a$ & 4.7 \\
& -16.3 & -16.1 & $15 s, 15 a$ & -16.6 & -16.5 & $17 s, 17 a$ & -16.4 \\
\hline
\end{tabular}

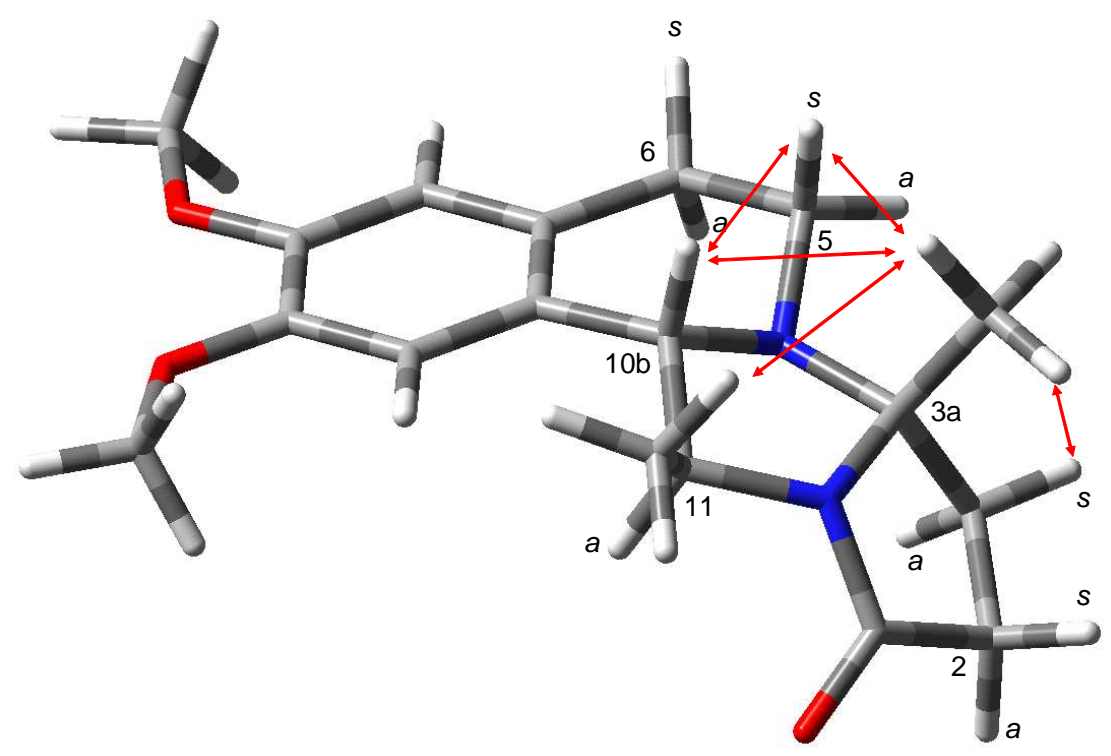

Figure 1. The preferred conformation, trans-B/C, of 11 in $\mathrm{CDCl}_{3}$ solution at $298 \mathrm{~K}$ as obtained from MM optimization with constraints from NMR spectroscopic analysis. Relevant NOE correlations are indicated with double-headed arrows. 
"small" (ca. $1 \mathrm{~Hz}$ ) limiting value. Using the $J$-data from Table 2 this allows for a rough estimate: 11 is ca. $85 \%$ trans-B/C, 13 and 17 are $80-85 \%$ cis-B/C and the rest are $90-100 \%$ cis-B/C. Thus, increasing the size of the substituent $\mathrm{R}$ or adding a condensed benzene ring (ring $\mathrm{E}$ ) on the lactam ring seems to shift the equilibrium towards the $c i s$-fused conformation. This is consistent with our previous findings on similar structures which lack the methyl substituent at ring C. ${ }^{5}$ Comparing the results, this methyl substitution in itself seems to cause a shift towards the cis$\mathrm{B} / \mathrm{C}$ conformation.

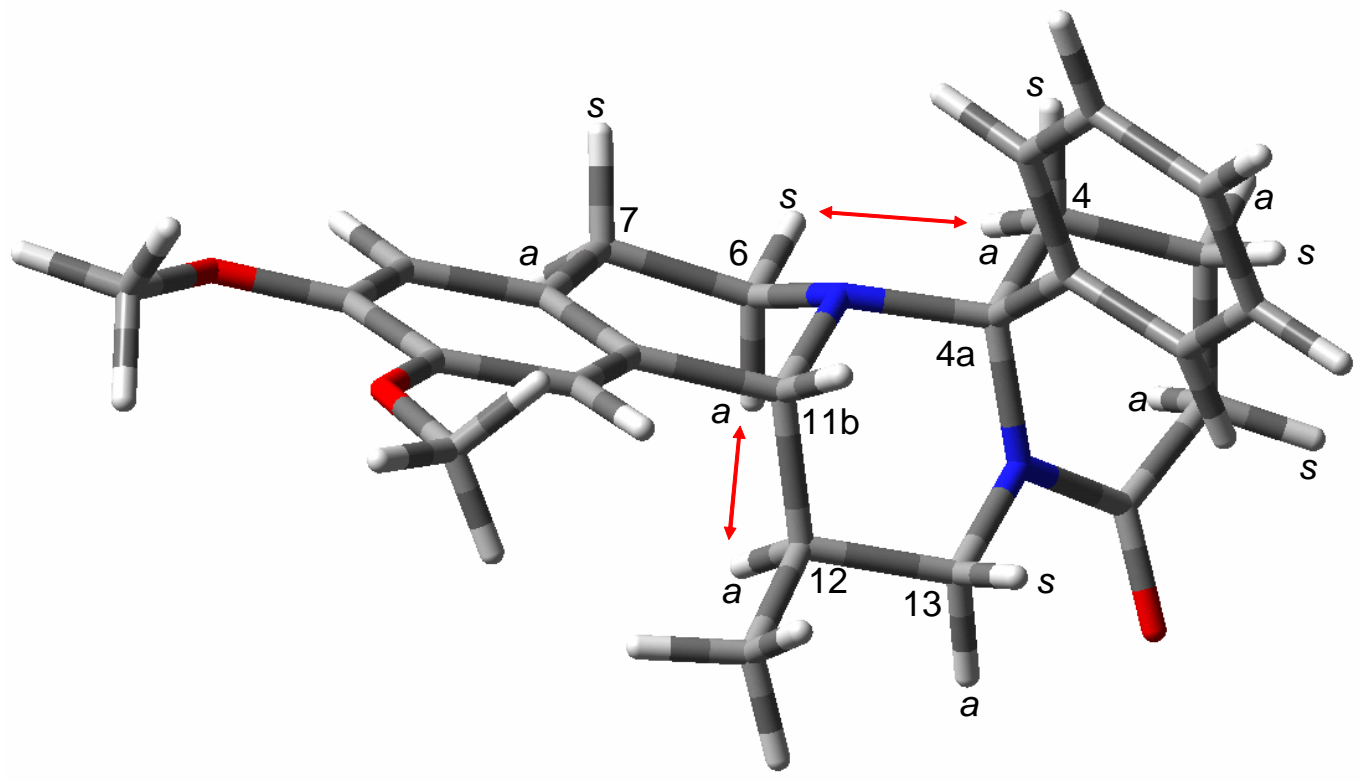

Figure 2. The preferred conformation, cis-B/C, of 16 in $\mathrm{CDCl}_{3}$ solution at $298 \mathrm{~K}$ as obtained from MM optimization with constraints from NMR spectroscopic analysis. Relevant NOE correlations are indicated with double-headed arrows.

The terminal six-membered piperidine ring in $\mathbf{1 5}$ and $\mathbf{1 6}$ (ring D) displayed large vicinal $\mathrm{H}, \mathrm{H}$-coupling constants within the anti-syn-anti sequence of its protons (i.e. ${ }^{3} J_{\mathrm{H}, \mathrm{H}}(2 a, 3 s)$ and ${ }^{3} J_{\mathrm{H}, \mathrm{H}}(3 s, 4 a)$ ), implying their trans-diaxial relationship. This is consistent with a chair or halfchair (with the amide group close to planar) conformation, with $\mathrm{R}$ in a (pseudo)axial position. When the terminal ring $\mathrm{D}$ is five-membered (pyrrolidine; derivatives 11-14) the $J$-coupling between the syn protons $(2 s, 3 s)$ is large, as is the coupling between the anti protons $(2 a, 3 a)$. Therefore, in the preferred conformation of this ring the syn hydrogens nearly eclipse each other (as do the anti hydrogens). For 11 and 12 (13 and 14), the eclipsed conformation is slightly opened so that the $2 s$ and $3 a$ ( $2 a$ and $3 s$ ) hydrogens become more antiperiplanar. The ortho protons of the phenyl group in phenyl-substituted $(\mathrm{R}=\mathrm{Ph})$ derivatives $\mathbf{1 6}$ and 20 were non- 
equivalent and heavily broadened at $298 \mathrm{~K}$, indicating that the rotation of this substituent is restricted and takes place in the slow-intermediate NMR time scale. The other phenylsubstituted compounds $(\mathbf{1 2}, \mathbf{1 4}, \mathbf{1 5}, \mathbf{1 8})$ did not show similar signal splitting. Apparently, there are steric 1,3-diaxial interactions present when the rings $\mathrm{C}$ and/or $\mathrm{D}$ are six-membered (hexahydropyrimidine and piperidine, respectively), which in case of $\mathbf{1 6}$ and $\mathbf{2 0}$ slow down the rotation of the phenyl substituent sufficiently to cause the observed ${ }^{1} \mathrm{H}$ signal splitting of the ortho protons.

Table 3. Carbon chemical shifts $\delta_{\mathrm{C}}$ (in ppm, $\mathrm{CDCl}_{3}, 298 \mathrm{~K}$ ) of 11-22. The assignment between shifts marked with a similar symbol is uncertain

\begin{tabular}{|c|c|c|c|c|c|c|c|}
\hline & 11 & 12 & 13 & 14 & & 15 & 16 \\
\hline $\mathrm{R}$ & $\mathrm{Me}$ & $\mathrm{Ph}$ & $\mathrm{Me}$ & $\mathrm{Ph}$ & $\mathrm{R}$ & $\mathrm{Ph}$ & $\mathrm{Ph}$ \\
\hline $\mathrm{n}$ & 0 & 0 & 1 & 1 & $\mathrm{n}$ & 0 & 1 \\
\hline 1 & 176.7 & 177.6 & 172.4 & 174.4 & 1 & 169.9 & 171.9 \\
\hline \multirow[t]{2}{*}{2} & 33.3 & 32.9 & 30.2 & 30.2 & 2 & 31.5 & 33.0 \\
\hline & & & & & 3 & 16.4 & 17.3 \\
\hline 3 & 33.3 & 30.1 & 31.3 & 33.0 & 4 & 32.3 & 36.6 \\
\hline $3 a$ & 83.9 & 92.0 & 78.3 & 84.4 & $4 a$ & 87.4 & 81.6 \\
\hline 5 & 42.0 & 41.0 & 38.8 & 37.6 & 6 & 41.4 & 37.6 \\
\hline 6 & 29.1 & 28.9 & 29.0 & 28.8 & 7 & 28.9 & 28.9 \\
\hline $6 a$ & $126.0^{\#}$ & $125.8^{*}$ & $126.6^{\dagger}$ & 126.1 & $7 \mathrm{a}$ & $125.7^{*}$ & 126.3 \\
\hline 7 & 111.9 & 111.2 & 111.4 & 111.3 & 8 & 111.1 & 111.2 \\
\hline 8 & $148.0 *$ & 148.0 & 148.1 & 148.1 & 9 & 148.0 & 148.0 \\
\hline 8-OMe & $56.0^{\dagger}$ & 55.8 & $55.8 *$ & 55.8 & 9-OMe & 55.8 & 55.8 \\
\hline 9 & $147.3^{*}$ & 147.3 & 146.1 & 145.9 & 10 & 147.2 & 145.9 \\
\hline 9-OMe & $55.9^{\dagger}$ & 55.9 & $56.1^{*}$ & 56.0 & $10-\mathrm{OMe}$ & 55.8 & 56.0 \\
\hline 10 & 107.4 & 109.2 & 112.3 & 112.0 & 11 & 109.6 & 112.0 \\
\hline $10 \mathrm{a}$ & $128.2^{\#}$ & $125.6^{*}$ & $127.7^{\dagger}$ & 127.8 & $11 \mathrm{a}$ & $125.7^{*}$ & 128.2 \\
\hline $10 \mathrm{~b}$ & 69.1 & 68.8 & 60.2 & 60.7 & $11 b$ & 63.7 & 60.1 \\
\hline 11 & 55.1 & 55.5 & 29.9 & 28.0 & 12 & 57.0 & 29.1 \\
\hline $11-\mathrm{Me}$ & 22.4 & 19.1 & 17.1 & 16.7 & 12-Me & 18.0 & 16.9 \\
\hline 12 & - & - & 42.9 & 43.9 & 13 & - & 45.3 \\
\hline \multirow[t]{4}{*}{$\mathrm{R}$} & 19.6 & i 145.3 & 23.4 & 144.5 & $\mathrm{R}$ & 144.5 & 144.2 \\
\hline & & $o 125.5$ & & 125.7 & & 127.2 & 129 (br) \\
\hline & & $m 128.4$ & & 129.2 & & 127.8 & 127.0 \\
\hline & & $p 127.6$ & & 127.4 & & 127.6 & 127.3 \\
\hline
\end{tabular}


Table 3. (Continued)

\begin{tabular}{|c|c|c|c|c|c|c|c|}
\hline & 17 & 18 & & 19 & 20 & & 22 \\
\hline $\mathrm{R}$ & $\mathrm{H}$ & $\mathrm{Ph}$ & $\mathrm{R}$ & $\mathrm{H}$ & $\mathrm{Ph}$ & $\mathrm{R}$ & $\mathrm{H}$ \\
\hline $\mathrm{n}$ & 0 & 0 & $\mathrm{n}$ & 1 & 1 & $\mathrm{n}$ & - \\
\hline 1 & 111.5 & 111.3 & 1 & 111.7 & 111.4 & 1 & 112.0 \\
\hline 2 & 148.2 & 148.1 & 2 & 148.4 & 148.2 & 2 & 148.6 \\
\hline 2-OMe & 55.8 & 55.9 & 2-OMe & 55.8 & 55.8 & 2-OMe & 55.9 \\
\hline 3 & 147.3 & 147.3 & 3 & 146.2 & 145.9 & 3 & 146.8 \\
\hline 3-OMe & 56.0 & 55.9 & 3-OMe & 56.2 & 56.0 & 3-OMe & 56.3 \\
\hline 4 & 108.9 & 109.3 & 4 & 112.2 & 112.2 & 4 & 113.1 \\
\hline $4 a$ & 125.6 & 125.9 & $4 a$ & 127.4 & 127.7 & $4 a$ & 126.3 \\
\hline $4 b$ & 73.1 & 70.9 & $4 \mathrm{~b}$ & 65.2 & 60.2 & $4 b$ & 61.5 \\
\hline 5 & 54.5 & 55.3 & 5 & 27.9 & 28.1 & $4 c$ & 124.3 \\
\hline \multirow[t]{5}{*}{ 5-Me } & 20.4 & 20.0 & $5-\mathrm{Me}$ & 16.8 & 16.7 & 5 & 128.0 \\
\hline & & & 6 & 45.6 & 43.2 & 6 & 124.0 \\
\hline & & & & & & 7 & 128.0 \\
\hline & & & & & & 8 & 118.7 \\
\hline & & & & & & $8 a$ & 140.2 \\
\hline 7 & 171.4 & 172.4 & 8 & 165.3 & 166.6 & 10 & 165.8 \\
\hline $7 a$ & 135.6 & 133.1 & $8 a$ & 133.8 & 131.0 & $10 \mathrm{a}$ & $133.6 *$ \\
\hline 8 & 124.3 & 124.3 & 9 & 123.9 & 124.1 & 11 & 124.0 \\
\hline 9 & 129.8 & 129.3 & 10 & 129.4 & 128.7 & 12 & 129.9 \\
\hline 10 & 132.1 & 132.4 & 11 & 131.7 & 132.0 & 13 & 132.6 \\
\hline 11 & 124.3 & 124.3 & 12 & 123.3 & 123.2 & 14 & 123.2 \\
\hline $11 \mathrm{a}$ & 140.5 & 145.8 & $12 \mathrm{a}$ & 140.8 & 147.5 & $14 \mathrm{a}$ & $135.2 *$ \\
\hline $11 b$ & 81.8 & 93.6 & $12 b$ & 76.5 & 84.5 & $14 \mathrm{~b}$ & 77.3 \\
\hline 13 & 41.3 & 42.8 & 14 & 36.4 & 38.6 & 16 & 37.4 \\
\hline 14 & 28.3 & 28.8 & 15 & 27.9 & 28.5 & 17 & 27.9 \\
\hline $14 \mathrm{a}$ & 125.8 & 125.9 & $15 \mathrm{a}$ & 126.1 & 126.3 & $17 \mathrm{a}$ & 126.5 \\
\hline \multirow[t]{4}{*}{$\mathrm{R}$} & - & $i 141.3$ & $\mathrm{R}$ & - & 139.6 & $\mathrm{R}$ & - \\
\hline & & o 125.9 & & & $126.1(\mathrm{br})$ & & \\
\hline & & $m 128.7$ & & & 129.4 & & \\
\hline & & $p 128.2$ & & & 128.1 & & \\
\hline
\end{tabular}

\section{Mass spectrometric fragmentations}

The primary fragmentations of compounds 11-20 and 22 are based on ring cleavages occurring in ring $\mathrm{C}$ (Scheme 5 and Table 4). The substitution $(\mathrm{H}, \mathrm{Me}$ or $\mathrm{Ph})$ at the position $3 \mathrm{a}(\mathbf{1 1}-\mathbf{1 4})$, $4 \mathrm{a}$ $(\mathbf{1 5}$ and 16), $11 \mathrm{~b}(\mathbf{1 7}, \mathbf{1 8}), 12 \mathrm{~b}(\mathbf{1 9}, \mathbf{2 0})$ or $14 \mathrm{~b}(\mathbf{2 2})$ as well as $n=0$ or 1 (Scheme 3) seems to have fairly decisive effect on the type of fragments obtained. For all compounds, ring $\mathrm{C}$ splits into relatively strong ion $\mathbf{a}+\mathbf{1}$ which is even the base peak when $\mathrm{n}=1$ and $\mathrm{R}=\mathrm{Me}$ or $\mathrm{Ph}$ and into $[\mathbf{M}-\mathbf{a}]^{+\bullet}$ which in turn is the base peak when $\mathrm{n}=0$ and $\mathrm{R}=\mathrm{H}$ or $\mathrm{Ph}(\mathbf{1 7}$ and 18). Furthermore, 11, 16, 19, 20 and 22 give some amount of the ion $\mathbf{a}-1$ which is the base peak for $\mathbf{1 9}(\mathrm{n}=1$ and 
$\mathrm{R}=\mathrm{Ph}$ ). The complementary ion $[\mathrm{M}-\mathbf{a}]^{+\bullet}$ is the base peak for $\mathbf{1 7}$ and $\mathbf{1 8}(\mathrm{n}=0$ and $\mathrm{R}=\mathrm{H}$ or $\mathrm{Ph}$ ) and also rather strong for the other compounds except 11 and 22. All compounds except 11, 13, 17 ( $\mathrm{n}=0,1,0$ and $\mathrm{R}=\mathrm{Me}, \mathrm{Me}$ and $\mathrm{H}$, respectively) and $\mathbf{2 2}$ ( $\mathrm{H}$ at $\mathrm{C}-14 \mathrm{~b})$ give relatively strong complementary ion $[\mathrm{M}-(\mathbf{a}+\mathbf{1})]^{+}$which is even the base peak for $\mathbf{1 4}$ and $\mathbf{2 0}(\mathrm{n}=1$ and $\mathrm{R}=\mathrm{Ph})$. Compounds 14, 19 and $20\left(\mathrm{n}=1 ; \mathrm{R}=\mathrm{Ph}, \mathrm{H}, \mathrm{Ph}\right.$, respectively) give also a weak ion $[\mathrm{M}-(\mathbf{a}-\mathbf{1})]^{+}$ and compounds $\mathbf{1 2}, \mathbf{1 4}$, and $\mathbf{1 5}(\mathrm{n}=0,1$ and 0 , respectively and $\mathrm{R}=\mathrm{Ph})$ a weak ion $[\mathrm{M}-(\mathbf{a}+\mathbf{2})]^{+\bullet}$. Ring $\mathrm{C}$ additionally splits into ion $\mathbf{b}$, which contains the $\mathrm{CH}_{3} \mathrm{CH}$-group (Scheme 5) more than ion $\mathbf{a}$. Ion $\mathbf{b}$ is the base peak for $\mathbf{1 2}(\mathrm{n}=0$ and $\mathrm{R}=\mathrm{Ph})$ and abundant for $\mathbf{1 5}(\mathrm{n}=0$ and $\mathrm{R}=\mathrm{Ph})$ and present also in the spectra of $\mathbf{1 7}$ and $\mathbf{1 8}(\mathrm{n}=0, \mathrm{R}=\mathrm{H}$ and $\mathrm{Ph})$. It is interesting that compounds 11, 15 and $19(\mathrm{n}=0$ and $1 ; \mathrm{R}=\mathrm{Me}, \mathrm{Ph}$ and $\mathrm{H}$, respectively) give the ion $\mathbf{b}-\mathbf{1}$ of which, however, only $\mathbf{1 5}(\mathrm{n}=0, \mathrm{R}=\mathrm{Ph})$ exhibited also the ion $\mathbf{b}$.

Table 4. Main fragments $[\mathrm{m} / \mathrm{z}(\mathrm{RA} \%)]$ from the studied compounds under electron ionization. b: $\mathrm{C}_{13} \mathrm{H}_{17} \mathrm{NO}_{2}^{+\bullet},(\mathbf{b}-\mathbf{1}): \mathrm{C}_{13} \mathrm{H}_{16} \mathrm{NO}_{2}^{+},(\mathbf{a}+\mathbf{1}): \mathrm{C}_{11} \mathrm{H}_{14} \mathrm{NO}_{2}^{+}, \mathbf{a}: \mathrm{C}_{11} \mathrm{H}_{13} \mathrm{NO}_{2}^{+\bullet},(\mathbf{a}-\mathbf{1}): \mathrm{C}_{11} \mathrm{H}_{12} \mathrm{NO}_{2}{ }^{+}$. The base peaks are written in italics

\begin{tabular}{|c|c|c|c|c|c|c|c|}
\hline Cmpd & $\mathrm{M}^{+\bullet}$ & {$[\mathrm{M}-\mathrm{H}]^{+}$} & {$\left[\mathrm{M}-\mathrm{CH}_{3}\right]^{+}$} & $\mathbf{b}$ & $(b-1)$ & $(\mathbf{a}+\mathbf{1})$ & $\begin{array}{l}\mathbf{a} \\
(\mathbf{a}-\mathbf{1})\end{array}$ \\
\hline $11^{\mathrm{a}}$ & $316(6)$ & $315(31)^{b}$ & & $219(16)$ & 218(39) & $192(100)^{\mathrm{c}}$ & $\begin{array}{l}191(3) \\
190(15)\end{array}$ \\
\hline & & & {$[\mathrm{M}-\mathrm{Ph}]^{+}$} & & & & - \\
\hline 12 & $378(1)$ & $377(1)$ & $\begin{array}{l}301(6) \\
{\left[\mathrm{M}-\mathrm{CH}_{3}\right]^{+}}\end{array}$ & $219(100)$ & - & $192(22)$ & $\begin{array}{l}- \\
191(5.5)\end{array}$ \\
\hline 13 & $330(3)$ & $329(0.4)$ & $\begin{array}{l}315(17) \\
{[\mathrm{M}-\mathrm{Ph}]^{+}}\end{array}$ & - & - & $192(100)$ & - \\
\hline 14 & $392(2)$ & - & $315(3)$ & - & - & $192(82)$ & $\begin{array}{l}- \\
-\end{array}$ \\
\hline 15 & $392(62)$ & 391(11) & $\begin{array}{l}315(100) \\
{[\mathrm{M}-\mathrm{Ph}]^{+}}\end{array}$ & $219(82)$ & $218(4)$ & $192(38)$ & $\begin{array}{l}- \\
191(11)\end{array}$ \\
\hline 16 & $406(14)$ & $405(0.9)$ & $329(8.5)$ & - & - & 192(100) & $\begin{array}{l}190(6) \\
-\end{array}$ \\
\hline 17 & $350(17)$ & $349(1)$ & - & $219(8)$ & - & $192(40)$ & $\begin{array}{l}- \\
191(5)\end{array}$ \\
\hline 18 & $426(24)$ & $425(0.7)$ & - & $219(33)^{\mathrm{d}}$ & ${ }^{-} \mathrm{C}_{13} \mathrm{H}_{17} \mathrm{NO}_{2}^{+}$ & 192(38) & 191(7) \\
\hline 19 & $364(26)$ & $363(3)$ & - & - & $218(7)$ & 192(9) & $\begin{array}{l}190(100) \\
191(5)\end{array}$ \\
\hline 20 & $440(2)$ & $439(0.4)$ & ${ }^{-}\left[\mathrm{M}-\mathrm{CH}_{3}\right]^{+}$ & - & - & 192(86) & $\begin{array}{l}190(9) \\
191(2)\end{array}$ \\
\hline 22 & $398(72.5)$ & $397(100)$ & $383(8)$ & $-{ }^{\mathrm{e}}$ & - & $192(20)$ & $190(21)$ \\
\hline
\end{tabular}

${ }^{\mathrm{a}} \mathrm{MS} / \mathrm{MS}$ 20.42-24.96 eV. ${ }^{\mathrm{b}}$ In fact $\left[\mathrm{M}+\mathrm{H}-\mathrm{H}_{2}\right]^{+} .{ }^{\mathrm{c}}$ Also $\mathbf{a}+\mathbf{2}: 193(17) .{ }^{\mathrm{d}}(\mathbf{b}+\mathbf{1}) 220(7) .{ }^{\mathrm{e}}(\mathbf{b}+\mathbf{1})$ 220(10). 
Table 4. (Continued)

\begin{tabular}{|c|c|c|c|c|c|c|}
\hline Cmpd & $\mathrm{C}_{13} \mathrm{H}_{9}{ }^{+}$ & $m / z 176$ & {$[\mathrm{M}-\mathbf{a}]^{+\bullet}$} & {$[\mathrm{M}-(\mathbf{a}+\mathbf{1})]^{+}$} & {$[\mathrm{M}-(\mathbf{a}-\mathbf{1})]^{+}$} & {$[\mathrm{M}-(\mathbf{a}+2)]^{+\bullet}$} \\
\hline \multirow{3}{*}{11} & & & $\mathrm{C}_{7} \mathrm{H}_{12} \mathrm{NO}^{+\bullet}$ & & & \\
\hline & - & {$\left[(\mathbf{b}-\mathbf{1})-\mathrm{C}_{3} \mathrm{H}_{6}\right]^{+}(94)$} & $126(35)$ & - & $f_{-}$ & - \\
\hline & & {$\left[\mathbf{b}-\mathrm{C}_{3} \mathrm{H}_{7}\right]^{+}$} & $\mathrm{C}_{12} \mathrm{H}_{13} \mathrm{NO}^{+\bullet}$ & $\mathrm{C}_{12} \mathrm{H}_{12} \mathrm{NO}^{+}$ & & $\mathrm{C}_{12} \mathrm{H}_{11} \mathrm{NO}^{+\bullet}$ \\
\hline \multirow[t]{2}{*}{12} & - & {$\left[\mathbf{a}-\mathrm{CH}_{3}\right]^{+}(3)$} & $187(44)$ & $186(52)$ & - & $185(9)$ \\
\hline & & & $\mathrm{C}_{8} \mathrm{H}_{13} \mathrm{NO}^{+\bullet}$ & & & \\
\hline \multirow[t]{2}{*}{13} & - & - & 139(18) & - & - & - \\
\hline & & & $\mathrm{C}_{13} \mathrm{H}_{14} \mathrm{NO}^{+}$ & $\mathrm{C}_{13} \mathrm{H}_{14} \mathrm{NO}^{+}$ & $\mathrm{C}_{13} \mathrm{H}_{16} \mathrm{NO}^{+}$ & $\mathrm{C}_{13} \mathrm{H}_{13} \mathrm{NO}^{+\bullet}$ \\
\hline \multirow[t]{2}{*}{14} & - & {$\left[\mathbf{a}-\mathrm{CH}_{3}\right]^{+}(5)$} & 201(47) & $200(100)$ & $202(8)$ & $199(9.5)$ \\
\hline & & {$\left[\mathbf{b}-\mathrm{C}_{3} \mathrm{H}_{7}\right]^{+}$} & $\mathrm{C}_{13} \mathrm{H}_{15} \mathrm{NO}^{+\bullet}$ & $\mathrm{C}_{13} \mathrm{H}_{14} \mathrm{NO}^{+}$ & & $\mathrm{C}_{13} \mathrm{H}_{13} \mathrm{NO}^{+\bullet}$ \\
\hline \multirow[t]{2}{*}{15} & - & {$\left[\mathbf{a}-\mathrm{CH}_{3}\right]^{+}(6)$} & 201(29) & $200(68)$ & - & 199(7) \\
\hline & & & $\mathrm{C}_{14} \mathrm{H}_{17} \mathrm{NO}^{+\bullet}$ & $\mathrm{C}_{14} \mathrm{H}_{16} \mathrm{NO}^{+}$ & & \\
\hline \multirow[t]{2}{*}{16} & - & {$\left[(\mathbf{b}-\mathbf{1})-\mathrm{C}_{3} \mathrm{H}_{6}\right]^{+}(10)$} & $215(56)$ & $214(79)$ & - & - \\
\hline & & & $\mathrm{C}_{10} \mathrm{H}_{9} \mathrm{NO}^{+\bullet}$ & & & \\
\hline \multirow[t]{2}{*}{17} & - & - & $159(100)$ & - & - & - \\
\hline & & & $\mathrm{C}_{16} \mathrm{H}_{13} \mathrm{NO}^{+\bullet}$ & $\mathrm{C}_{16} \mathrm{H}_{12} \mathrm{NO}^{+}$ & & \\
\hline \multirow[t]{2}{*}{18} & $165(28)$ & - & $235(100)$ & $234(65)$ & - & - \\
\hline & & & $\mathrm{C}_{11} \mathrm{H}_{11} \mathrm{NO}^{+\bullet}$ & $\mathrm{C}_{11} \mathrm{H}_{10} \mathrm{NO}^{+}$ & $\mathrm{C}_{11} \mathrm{H}_{12} \mathrm{NO}^{+}$ & \\
\hline \multirow[t]{2}{*}{19} & - & {$\left[(\mathbf{b}-\mathbf{1})-\mathrm{C}_{3} \mathrm{H}_{6}\right]^{+}(8)$} & $173(74)$ & $172(5)$ & $174(8)^{\mathrm{g}, \mathrm{h}}$ & - \\
\hline & & & $\mathrm{C}_{17} \mathrm{H}_{15} \mathrm{NO}^{+\bullet}$ & $\mathrm{C}_{17} \mathrm{H}_{14} \mathrm{NO}^{+}$ & & \\
\hline \multirow[t]{2}{*}{20} & $165(16.5)$ & {$\left[(\mathbf{b}-\mathbf{1})-\mathrm{C}_{3} \mathrm{H}_{6}\right]^{+}(7)$} & $249(80)$ & $248(100)$ & - & - \\
\hline & & & $\mathrm{C}_{14} \mathrm{H}_{9} \mathrm{NO}^{+\bullet}$ & & $\mathrm{C}_{14} \mathrm{H}_{10} \mathrm{NO}^{+}$ & \\
\hline 22 & $165(30)$ & - & $207(5)$ & - & 208(19) & - \\
\hline
\end{tabular}

${ }^{\mathrm{f}}$ Gives also the ion $\mathrm{C}_{11} \mathrm{H}_{12} \mathrm{NO}^{+}: 174(13) .{ }^{\mathrm{g}}$ Contains $1 / 9$ of $\mathrm{C}_{10} \mathrm{H}_{8} \mathrm{NO}_{2}{ }^{+} .{ }^{\mathrm{h}}[\mathrm{M}-(\mathbf{a}-\mathbf{2})]^{+\bullet}: \mathrm{C}_{11} \mathrm{H}_{13} \mathrm{NO}^{+\bullet}$ 175(6); contains $1 / 6$ of $\mathrm{C}_{10} \mathrm{H}_{9} \mathrm{NO}_{2}{ }^{+\bullet}$.
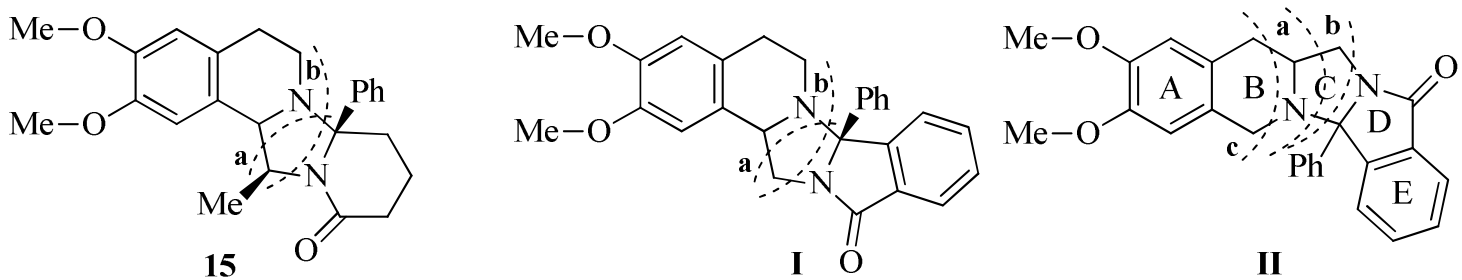

Scheme 5. The primary fragmentation routes for linearly-condensed compounds (15 as an example) studied in this work in comparison with the major fragmentations (I and II) of the polycyclic lactams reported earlier. ${ }^{5 \mathrm{~b}}$

All compounds except 13, 17, 18 and 22 give also some amount of the ion $m / z, 176$ which for 11, 16, 18 and $20(\mathrm{n}=0,1,0$ and $1 ; \mathrm{R}=\mathrm{Me}, \mathrm{Ph}, \mathrm{Ph}$ and $\mathrm{Ph}$, respectively) corresponds to $\left[(\mathbf{b}-1)-\mathrm{C}_{3} \mathrm{H}_{6}\right]^{+}$whereas 12 and $\mathbf{1 5}(\mathrm{n}=0$ and $\mathrm{R}=\mathrm{Ph})$ exhibiting a strong ion $\mathbf{b}$, give the ion 
$\left[\mathbf{b}-\mathrm{C}_{3} \mathrm{H}_{7}\right]^{+}$or $\left[\mathrm{M}-\mathbf{a}-\mathrm{CH}_{3}\right]^{+}$instead. For $\mathbf{1 4}(\mathrm{n}=1, \mathrm{R}=\mathrm{Ph}) \mathrm{m} / z 176$ corresponds to ion $\left[\mathbf{a}-\mathrm{CH}_{3}\right]^{+}$. For 11 and 15 for which $\mathrm{n}=0$ and $\mathrm{R}=\mathrm{Me}$ or $\mathrm{Ph}$ (Scheme 3), $\left[\mathrm{M}-\mathrm{CH}_{3}\right]^{+}$is the base peak and also $13(\mathrm{n}=1, \mathrm{R}=\mathrm{Me})$ and $22(\mathrm{H}$ at $\mathrm{C}-4 \mathrm{~b})$ give a small amount of $\left[\mathrm{M}-\mathrm{CH}_{3}\right]^{+}$ion whereas 12 (n $=0, \mathrm{R}=\mathrm{Ph}), \mathbf{1 4}(\mathrm{n}=1, \mathrm{R}=\mathrm{Ph})$ and $\mathbf{1 6}(\mathrm{n}=1, \mathrm{R}=\mathrm{Ph})$ give a fairly weak ion $[\mathrm{M}-\mathrm{Ph}]^{+}$instead.

The hexacyclic lactam is greatly stabilized by the benzo-fusions which is proved by the fact that its base peak is $[\mathrm{M}-\mathrm{H}]^{+}$and also the molecular ion is very strong $(72 \%)$. This is on line with the observation that otherwise it gives a great number of medium or weak ions (Tables 4 and 5). As to the other compounds $11,13,14,17$ and 18 give relatively few and mainly low mass fragments whereas compounds 12, 15, 16, 19, and 20 give rather many further fragments (Table 5). The common features for the first-mentioned group seem to be that $n=0(\mathbf{1 1}, \mathbf{1 7}$ and 18) or $n=1$ and $\mathrm{R}=\mathrm{Me}$ or $\mathrm{Ph}(\mathbf{1 3}$ and 14). As to the second group the common features are $\mathrm{n}=0$ or 1 and $\mathrm{R}=$ $\mathrm{Ph}(12,15,16$ and 20), for $19 \mathrm{n}=1$ and $\mathrm{R}=\mathrm{H}$. If we compare this situation with the groups in Scheme 1 12, 17 and 18 ( $\mathrm{n}=0$ and $\mathrm{R}=\mathrm{Ph}$ ) fall out from these groups probably due to the fivemembered ring in $\mathbf{1 2}$ and the benzo-fusion (and $\mathrm{n}=1$ ) in $\mathbf{1 7}$ and 18. On the other hand 15, 16, 19 and 20 consist of a six-membered oxo-ring $+\mathrm{R}=\mathrm{Ph}(\mathbf{1 5}$ and 16) and a benzo-fusion with $\mathrm{n}=1$ (19 and 20).

Table 5. Other ions $(\mathrm{m} / \mathrm{z}[\mathrm{RA} \approx 5 \%])$

\begin{tabular}{|c|c|}
\hline Cmpd & \\
\hline 11 & {$[\mathrm{M}+\mathrm{H}-\mathbf{b}]^{+\bullet}=\mathrm{C}_{5} \mathrm{H}_{8} \mathrm{NO}^{+\bullet}: 98(14)^{\mathrm{a}}$} \\
\hline 12 & $\begin{array}{l}\mathrm{C}_{11} \mathrm{H}_{13} \mathrm{~N}^{+\bullet}: 159(8) ; \mathrm{C}_{11} \mathrm{H}_{12} \mathrm{~N}^{+}: 158(5) ; \mathrm{C}_{10} \mathrm{H}_{10} \mathrm{~N}^{+}: 144(12) ; \mathrm{C}_{9} \mathrm{H}_{9} \mathrm{~N}^{+\bullet}: 131(21) ; \mathrm{C}_{9} \mathrm{H}_{8} \mathrm{~N}^{+}: \\
130(7) ; \mathrm{C}_{8} \mathrm{H}_{7} \mathrm{~N}^{+\bullet}+\mathrm{C}_{9} \mathrm{H}_{9}^{+}(1: 1): 117(8) ; \mathrm{C}_{7} \mathrm{H}_{6} \mathrm{~N}^{+}+\mathrm{C}_{8} \mathrm{H}_{8}^{+\bullet}(6: 1): 104(10) ; \mathrm{C}_{7} \mathrm{H}_{5} \mathrm{~N}^{+\bullet}+\mathrm{C}_{8} \mathrm{H}_{7}^{+} \\
(1: 1): 103(11) ; 56(5)\end{array}$ \\
\hline 13 & $\mathrm{C}_{7} \mathrm{H}_{10} \mathrm{~N}^{+}: 124(21) ; \mathrm{C}_{6} \mathrm{H}_{9} \mathrm{NO}^{+\bullet}: 111(22.5) ; \mathrm{C}_{5} \mathrm{H}_{8} \mathrm{NO}^{+}:$98(41); 55(6), 56(6), 42(7) \\
\hline 14 & $\mathrm{C}_{10} \mathrm{H}_{10} \mathrm{NO}^{+}: 160(9.5) ; \mathrm{C}_{11} \mathrm{H}_{12} \mathrm{~N}^{+}: 158(6) ; 117(7) ; \mathrm{C}_{7} \mathrm{H}_{6} \mathrm{~N}^{+}: 104(6) ; 103(5) ; 56(6)$ \\
\hline 15 & $\begin{array}{l}{\left[\mathrm{M}-\mathrm{C}_{2} \mathrm{H}_{5}\right]^{+}: 363(19) ;\left[\mathrm{M}-\mathrm{C}_{4} \mathrm{H}_{7} \mathrm{O}\right]^{+}: 321(13) ;\left[\mathrm{M}-\mathrm{C}_{7} \mathrm{H}_{9}\right]^{+}: 299(11) ;\left[\mathrm{M}-\mathrm{C}_{5} \mathrm{H}_{8} \mathrm{NO}\right]^{+}:} \\
294(30) ; \mathrm{C}_{12} \mathrm{H}_{14} \mathrm{~N}^{+}: 172(25) ; \mathrm{C}_{11} \mathrm{H}_{12} \mathrm{~N}^{+}: 158(27.5) ; \mathrm{C}_{9} \mathrm{H}_{9} \mathrm{~N}^{+\bullet}: 131(16) ; \mathrm{C}_{9} \mathrm{H}_{8} \mathrm{~N}^{+}: 130(10) ; \\
104(14) ; 103(20) ; 91(5) ; 77(7), 55(12)\end{array}$ \\
\hline 16 & $\begin{array}{l}{\left[\mathrm{M}-\mathrm{C}_{7} \mathrm{H}_{7}\right]^{+}: 315(11) ; \mathrm{C}_{13} \mathrm{H}_{14} \mathrm{NO}^{+}: 200(12) ; 186(5), 174(5) ; 145(29), 130(8), 117(8),} \\
103(7) ; 69(6) ; 58(7) ; 55(6.5) ; 43(19) ; 42(7)\end{array}$ \\
\hline 17 & $130(21) ; 90(18)$ \\
\hline 18 & $\mathrm{C}_{15} \mathrm{H}_{12} \mathrm{~N}^{+}: 206(16) ; 58(5) ; 43(15)$ \\
\hline 19 & $\begin{array}{l}\mathrm{C}_{10} \mathrm{H}_{8} \mathrm{NO}^{+}: 158(12) ; 146(6) ; 145(4) ; 144(5) ; \mathrm{C}_{8} \mathrm{H}_{6} \mathrm{NO}^{+}: 132(29) ; 130(6) ; \mathrm{C}_{8} \mathrm{H}_{7} \mathrm{~N}^{+\bullet}: \\
117(11) ; \mathrm{C}_{7} \mathrm{H}_{4} \mathrm{O}^{+\bullet}+\mathrm{C}_{7} \mathrm{H}_{6} \mathrm{~N}^{+}(3: 1): 104(13) ; 103(5) ; 90(5) ; 77(5) ; 43(10) ; 41(6)\end{array}$ \\
\hline 20 & $\begin{array}{l}\mathrm{C}_{16} \mathrm{H}_{12} \mathrm{NO}^{+}: 234(6) ; \mathrm{C}_{16} \mathrm{H}_{14} \mathrm{~N}^{+}+\mathrm{C}_{15} \mathrm{H}_{10} \mathrm{NO}^{+}(3: 1): 220(14) ; \mathrm{C}_{14} \mathrm{H}_{10} \mathrm{NO}^{+}: 208(8) ; \\
\mathrm{C}_{14} \mathrm{H}_{8} \mathrm{NO}^{+}+\mathrm{C}_{15} \mathrm{H}_{12} \mathrm{~N}^{+}(4: 1): 206(8) ; \mathrm{C}_{13} \mathrm{H}_{9} \mathrm{~N}^{+\bullet}: 179(6) ; 130(8) ; 104(6) ; 58(10) ; 43(33)\end{array}$ \\
\hline 22 & $\begin{array}{l}\left(\left[\mathrm{M}-\mathrm{C}_{2} \mathrm{H}_{5}\right]^{+}+[\mathrm{M}-\mathrm{CHO}]^{+}\right): 369(20) ; 301(10) ; \mathrm{C}_{16} \mathrm{H}_{12} \mathrm{~N}_{2} \mathrm{O}^{+\bullet}: 248(5) ; \mathrm{C}_{16} \mathrm{H}_{11} \mathrm{~N}_{2} \mathrm{O}^{+}: \\
247(5) ; \mathrm{C}_{15} \mathrm{H}_{9} \mathrm{~N}_{2} \mathrm{O}_{3}^{+}: 233(12) ; \mathrm{C}_{12} \mathrm{H}_{12} \mathrm{NO}^{+}: 186(24) ; \mathrm{C}_{10} \mathrm{H}_{13} \mathrm{NO}_{2}^{+\bullet}+\mathrm{C}_{13} \mathrm{H}_{9} \mathrm{~N}^{+\bullet}(1: 1): \\
179(17) ; \mathrm{C}_{10} \mathrm{H}_{12} \mathrm{NO}_{2}^{+}+\mathrm{C}_{13} \mathrm{H}_{8} \mathrm{~N}^{+}(2: 5): 178(8) ; 77(12) ; 69(5) ; 57(7) ; 44(14) ; 43(7) ; \\
\text { 41(6);40(11) }\end{array}$ \\
\hline
\end{tabular}

${ }^{\mathrm{a}} \mathrm{MS} / \mathrm{MS}$ from $[\mathrm{M}+\mathrm{H}]^{+}$at $33.57-41.03 \mathrm{eV}$. For the other results at this voltage see Experimental, compound 11. 


\section{Conclusions}

Our results demonstrate that 1-substituted 1,2,3,4-tetrahydroisoquinoline 1,2- and 1,3-diamines, bearing a methyl substituent or an aromatic ring in the side chain, were conveniently transformed to tetra-, penta- and hexacyclic lactams by domino cyclocondensations with acyclic or aromatic $\gamma$ - or $\delta$-oxo acids. The NMR analyses proved that each cyclocondensations took place with practically full diastereoselectivity ( $d e \sim 100 \%$ ) in favour of the cis isomer. Compound 11 prefers a conformation with a trans-B/C ring fusion, whereas the other derivatives $(\mathbf{1 2 - 2 0}, \mathbf{2 2})$ favour cis-B/C fusion. These conformers can change into each other through a ring-inversion of ring $\mathrm{B}$ combined with an umbrella inversion of its nitrogen atom, but the population of the preferred conformer is always $80-100 \%$. Increasing the size of substituent R, or adding a condensed benzene ring at the lactam ring, shifts the conformational equilibrium towards the cis-B/C conformer.

\section{Experimental Section}

General. Melting points were measured on a Kofler hot-plate microscope apparatus and are uncorrected. Column chromatography was performed with silica gel 60 (0.063-0.200). For routine thin-layer chromatography (TLC), Silica gel $60 \mathrm{~F}_{254}$ plates (Merck, Germany) were used. Elemental analyses were performed with a Perkin-Elmer 2400 CHNS elemental analyser. Compounds $\mathbf{1 0}^{7}$ and $\mathbf{2 1}^{8}$ were prepared according to known procedures.

\section{NMR spectra}

The NMR spectra were recorded in $\mathrm{CDCl}_{3}$, DMSO- $\mathrm{d}_{6}$ or $\mathrm{D}_{2} \mathrm{O}$ solutions on JEOL JNM-LA400, Bruker AVANCE DRX 400 and AVANCE 500 spectrometers. Chemical shifts are given in $\delta$ $(\mathrm{ppm})$ relative to TMS $\left(\mathrm{CDCl}_{3}\right.$ and DMSO- $\left.\mathrm{d}_{6}\right)$ or to TSP $\left(\mathrm{D}_{2} \mathrm{O}\right)$ as internal standards. The spectra of the products were acquired without sample spinning at $298 \mathrm{~K}$. The NMR experiments consisted of standard ${ }^{1} \mathrm{H}$ NMR (using a $30^{\circ}$ flip angle and a $5 \mathrm{~s}$ pulse repetition time), ${ }^{13} \mathrm{C}$ NMR with broad-band proton decoupling, dqf-COSY, 1D and 2D NOESY (with a mixing time of 0.3 s), multiplicity-edited HSQC (optimized for a one-bond coupling of $145 \mathrm{~Hz}$ and set to show $\mathrm{CH}$ and $\mathrm{CH}_{3}$ signals positive and $\mathrm{CH}_{2}$ signals negative), and $\mathrm{HMBC}$ (optimized for long-range couplings of $8 \mathrm{~Hz}$ with a low-pass $J$-filter optimized to remove signals due to one-bond coupling around $145 \mathrm{~Hz}$ ) measurements. Proton chemical shifts $\delta_{\mathrm{H}}$ and proton-proton coupling constants $J_{\mathrm{H}, \mathrm{H}}$ were extracted by using the spectral simulation and analysis tool PER included in the PERCH NMR software package (version 2008.1). ${ }^{12}$ The initial guess for the NMR parameters was obtained manually from the ${ }^{1} \mathrm{H}$ spectra or by trial-and-error in case of crowded/complicated spectral regions, which was then refined iteratively by using the integral-transform and total lineshape-fitting modes of the software. Manual adjustment and iterative fitting of parameters was repeated until good visual comparison was achieved between the calculated and the observed 
spectra. Structural models were obtained from molecular-mechanics modelling (MM+ force field) by using the HyperChem 7.0 software. Distance and torsion angle restraints (with the software's default force constants) were applied in the initial modelling based on the observed NOE and $J$-coupling constant data, and when a satisfactory structure was obtained it was refined without restraints to yield the final model.

\section{Mass spectral measurements}

The electron ionization mass spectra (Tables 4 and 5) were recorded on a VG ZABSpec mass spectrometer (VG Analytical, Division of Fisons, Manchester, UK) equipped with the Opus V3.3X program package (Fisons Instruments, Manchester, UK). The ionization energy used was $70 \mathrm{eV}$ and the source temperature was $160{ }^{\circ} \mathrm{C}$. The accelerating voltage was $8 \mathrm{kV}$ and the trap current was $200 \mathrm{~mA}$. Perfluorokerosene was used to calibrate the mass scale. A small amount of solid sample dissolved in $\mathrm{MeOH}$ was placed in a capillary tube and the solvent was evaporated off with hot air. Thereafter, the sample was introduced into the ionization chamber via the solid inlet. The fragmentation pathways were confirmed by linked scans at constant $\mathrm{B} / \mathrm{E}$ or $\mathrm{B}^{2} / \mathrm{E}$ (first field-free region, FFR1) without collision gas. The low-resolution $\mathrm{B} / \mathrm{E}$ and $\mathrm{B}^{2} / \mathrm{E}$ spectra were measured with a resolving power of 3000 (10\% valley definition). The accurate masses were determined by voltage scanning with a resolving power of $6000-10,000$. The compositions of the molecular ions based on their accurate masses are given in Table 6.

For the low-resolution spectra, consecutive scans selected from the stable and constant part of the total ion current chromatogram were averaged to obtain more reproducible abundances. For accurate masses and linked scans, $\geq 10$ scans were averaged to minimize noise and to eliminate random peaks.

Since our VG ZABSpec was taken out of use, the mass spectrum of $\mathbf{1 1}$ was measured on a Bruker micrOTOF-Q ESI-HRMS instrument (Bruker Daltonics, Bremen, Germany). Mass spectrometer was controlled by Compass 1.3 for micrOTOF software package (Bruker Daltonics). The sample of $\mathbf{1 1}$ was dissolved in HPLC-grade acetonitrile (VWR International, Leuven, Belgium). The sample was then introduced to source using infusion pump. Positive ionization mode was used for MS analysis. The capillary voltage was maintained at $-4500 \mathrm{~V}$ and the end plate offset at $-500 \mathrm{~V}$. Nitrogen was used as nebulizer and drying gas. The pressure for the nebuliser gas was set at 0.4 bar. The drying gas flow rate was $4.0 \mathrm{~L} / \mathrm{min}$ and the drying gas temperature $200^{\circ} \mathrm{C}$. Mass detection was performed in the $\mathrm{m} / \mathrm{z}$ range 50-1500. The resolving power of the mass spectrometer was typically 8000-12000. Sodium formate clusters were used for external calibration of the $\mathrm{m} / \mathrm{z}$ range. Compass DataAnalysis 4.0 (Bruker Daltonics) was used for interpreting the mass data.

For collision induced dissociation (CID) MS/MS measurements, the collision energy in the $\mathrm{m} / \mathrm{z}$ range 50-1500 varied from $20 \mathrm{eV}$ or from $30 \mathrm{eV}(\mathrm{m} / \mathrm{z} 50)$ to $75 \mathrm{eV}(\mathrm{m} / \mathrm{z} 1500)$. Argon was used as collision gas. The mass spectrometer was operated in data-dependent mode to automatically select the most abundant precursor ions. 
Table 6. Composition of the molecular ions

\begin{tabular}{llllllll}
\hline & $\mathrm{M}^{+\bullet}$ & Calcd & Obsd & & $\mathrm{M}^{+\bullet}$ & Calcd & Obsd \\
\hline $\mathbf{1 1}$ & $\mathrm{C}_{18} \mathrm{H}_{24} \mathrm{~N}_{2} \mathrm{O}_{3}^{+\bullet}$ & 316.1787 & 316.1780 & $\mathbf{1 7}$ & $\mathrm{C}_{21} \mathrm{H}_{22} \mathrm{~N}_{2} \mathrm{O}_{3}^{+\bullet}$ & 350.1630 & 350.1631 \\
$\mathbf{1 3}$ & $\mathrm{C}_{19} \mathrm{H}_{26} \mathrm{~N}_{2} \mathrm{O}_{3}^{+\bullet}$ & 330.1943 & 330.1938 & $\mathbf{1 8}$ & $\mathrm{C}_{27} \mathrm{H}_{26} \mathrm{~N}_{2} \mathrm{O}_{3}^{+\bullet}$ & 426.1943 & 426.1940 \\
$\mathbf{1 4}$ & $\mathrm{C}_{24} \mathrm{H}_{28} \mathrm{~N}_{2} \mathrm{O}_{3}^{+\bullet}$ & 392.2100 & 392.2104 & $\mathbf{1 9}$ & $\mathrm{C}_{22} \mathrm{H}_{24} \mathrm{~N}_{2} \mathrm{O}_{3}^{+\bullet}$ & 364.1790 & 364.1781 \\
$\mathbf{1 2}$ & $\mathrm{C}_{23} \mathrm{H}_{26} \mathrm{~N}_{2} \mathrm{O}_{3}^{+\bullet}$ & 378.1943 & 378.1931 & $\mathbf{2 0}$ & $\mathrm{C}_{28} \mathrm{H}_{28} \mathrm{~N}_{2} \mathrm{O}_{3}^{+\bullet}$ & 440.2100 & 440.2093 \\
$\mathbf{1 5}$ & $\mathrm{C}_{23} \mathrm{H}_{26} \mathrm{~N}_{2} \mathrm{O}_{3}^{+\bullet}$ & 392.2100 & 392.2102 & $\mathbf{2 2}$ & $\mathrm{C}_{25} \mathrm{H}_{22} \mathrm{~N}_{2} \mathrm{O}_{3}^{+\bullet}$ & 398.1630 & 398.1612 \\
$\mathbf{1 6}$ & $\mathrm{C}_{25} \mathrm{H}_{30} \mathrm{~N}_{2} \mathrm{O}_{3}^{+\bullet}$ & 406.2256 & 406.2248 & & & & \\
\hline
\end{tabular}

\section{Preparation of the starting materials}

\section{2-Benzyloxycarbonylamino- $N$-[2-(3,4-dimethoxyphenyl)ethyl]propanamide (6)}

To a stirred and ice-salt bath-cooled solution of $N$-benzyloxycarbonyl-DL-alanine $(5,11.16 \mathrm{~g}$, $0.05 \mathrm{~mol})$ and triethylamine $(5.06 \mathrm{~g}, 0.05 \mathrm{~mol})$ in anhydrous toluene $(150 \mathrm{~mL})$, ethyl chloroformate $(5.43 \mathrm{~g}, 0.05 \mathrm{~mol})$ was added dropwise at a rate low enough to keep the internal temperature below $-10{ }^{\circ} \mathrm{C}$. After $5 \mathrm{~min}$, a solution of homoveratrylamine $(9.06 \mathrm{~g}, 0.05 \mathrm{~mol})$ in $\mathrm{CH}_{2} \mathrm{Cl}_{2}(50 \mathrm{~mL})$ was added dropwise, the internal temperature being kept below $0{ }^{\circ} \mathrm{C}$. When the addition was complete, the reaction mixture was heated under reflux for $5 \mathrm{~min}$. The mixture was allowed to cool down to room temperature and $\mathrm{CHCl}_{3}(250 \mathrm{~mL})$ was added. The mixture was next washed with saturated $\mathrm{NaHCO}_{3}$ solution $(3 \times 75 \mathrm{~mL})$ and water $(2 \times 75 \mathrm{~mL})$, and then dried $\left(\mathrm{Na}_{2} \mathrm{SO}_{4}\right)$, and the solvent was removed in vacuo to give a crude oily product, which crystallized on treatment with $\mathrm{Et}_{2} \mathrm{O}$. The crystals were filtered off, washed with $\mathrm{Et}_{2} \mathrm{O}$ and recrystallized from EtOAc.

White crystalline substance, yield: $15.30 \mathrm{~g}(79 \%)$; mp 126-128 ${ }^{\circ} \mathrm{C} .{ }^{1} \mathrm{H}$ NMR (400 $\left.\mathrm{MHz}, \mathrm{CDCl}_{3}\right)$ : $\delta_{\mathrm{H}} 1.34\left(3 \mathrm{H}, \mathrm{d}, \mathrm{J}=7.0 \mathrm{~Hz}, \mathrm{CH}_{3}\right), 2.66-2.77\left(2 \mathrm{H}, \mathrm{m}, \mathrm{ArCH}_{2}\right), 3.36-3.54\left(2 \mathrm{H}, \mathrm{m}, \mathrm{NCH}_{2}\right), 3.84(3 \mathrm{H}$, s, $\left.\mathrm{OCH}_{3}\right), 3.86\left(3 \mathrm{H}, \mathrm{s}, \mathrm{OCH}_{3}\right), 4.08-4.18(\mathrm{~m}, 1 \mathrm{H}, \mathrm{NCH}), 5.08\left(2 \mathrm{H}, \mathrm{dd}, \mathrm{OCH}_{2}, J=18,6,12,2 \mathrm{~Hz}\right)$, $5.30(1 \mathrm{H}$, br s, $\mathrm{NH}), 6.09(1 \mathrm{H}$, br s, $\mathrm{NH}), 6,70\left(2 \mathrm{H}\right.$, br s, $\left.\mathrm{C}_{6} H_{3}\right), 6.79\left(1 \mathrm{H}, \mathrm{d}, J=8,3 \mathrm{~Hz}, \mathrm{C}_{6} H_{3}\right)$, 7.28-7.38 (5H, m, $\left.\mathrm{C}_{6} H_{5}\right) .{ }^{13} \mathrm{C} \mathrm{NMR}\left(100 \mathrm{MHz}, \mathrm{CDCl}_{3}\right): \delta_{\mathrm{C}} 19.1,35.6,41.3,51.0,56.2,56.3$, 67.3, 111.9, 112.5, 121.1, 128.4, 128.6, 128.9, 131.8, 136.6, 148.1, 149.5, 156.4, 172.9. Anal. Calcd. for $\mathrm{C}_{21} \mathrm{H}_{26} \mathrm{~N}_{2} \mathrm{O}_{5} \mathrm{C}, 65.27 ; \mathrm{H}, 6.78 ; \mathrm{N}, 7.25$; Found C, 65.02; H, 6.49; N, 7.13\%.

\section{1-[1'-(Benzyloxycarbonylamino)ethyl]-6,7-dimethoxy-3,4-dihydroisoquinoline (7)}

To a stirred solution of the amide $6(15.00 \mathrm{~g}, 38.8 \mathrm{mmol})$ in dry $\mathrm{CHCl}_{3}(300 \mathrm{~mL}), \mathrm{POCl}_{3}(18.00$ $\mathrm{g}, 117.4 \mathrm{mmol}$ ) was added. The mixture was heated under reflux for $3 \mathrm{~h}$, and then evaporated in vacuo. The oily residue was dissolved in water $(250 \mathrm{~mL})$ under gentle warming, and the solution was cooled and extracted with EtOAc $(2 \times 75 \mathrm{~mL})$. The aqueous phase was made alkaline with $25 \% \mathrm{NaOH}$ solution with cooling, and extracted with $\mathrm{CHCl}_{3}(4 \times 150 \mathrm{~mL})$. The combined organic extracts were dried $\left(\mathrm{Na}_{2} \mathrm{SO}_{4}\right)$ and evaporated to give an oily product, which crystallized on treatment with $\mathrm{Et}_{2} \mathrm{O}$. The crystalline product was filtered off, washed with $\mathrm{Et}_{2} \mathrm{O}$ and used in the next step without further purification.

Yield: $10.69 \mathrm{~g}(75 \%)$. An analytical sample of 7 was recrystallized from $i \operatorname{Pr}_{2} \mathrm{O}$ to give beige crystals, mp 88-89 ${ }^{\circ} \mathrm{C} .{ }^{1} \mathrm{H} \mathrm{NMR}\left(400 \mathrm{MHz}, \mathrm{CDCl}_{3}\right): \delta_{\mathrm{H}} 1.39\left(3 \mathrm{H}, \mathrm{d}, J=6.6 \mathrm{~Hz}, \mathrm{CH}_{3}\right), 2.52-2.70$ 
$\left(2 \mathrm{H}, \mathrm{m}, \mathrm{ArCH}_{2}\right), 3.33-3.47\left(1 \mathrm{H}, \mathrm{m}, \mathrm{NCH}_{2}\right), 3.78-3.95\left(7 \mathrm{H}, \mathrm{m}, \mathrm{NCH}_{2}, 2 \times \mathrm{OCH}_{3}\right), 4.95-5.04(1 \mathrm{H}$, m, $\left.\mathrm{NCH}_{2}\right), 5.14\left(2 \mathrm{H}, \mathrm{dd}, J=15.4,12.3 \mathrm{~Hz}, \mathrm{OCH}_{2}\right), 6.47(1 \mathrm{H}, \mathrm{d}, J=6.4 \mathrm{~Hz}, \mathrm{NH}), 6.71(1 \mathrm{H}, \mathrm{s}$, $\left.\mathrm{C}_{6} \mathrm{H}_{2}\right), 6.98\left(1 \mathrm{H}, \mathrm{s}, \mathrm{C}_{6} \mathrm{H}_{2}\right), 7.27-7.38\left(5 \mathrm{H}, \mathrm{m}, \mathrm{C}_{6} H_{5}\right) .{ }^{13} \mathrm{C} \mathrm{NMR}\left(100 \mathrm{MHz}, \mathrm{CDCl}_{3}\right): \delta_{\mathrm{C}} 21.6,26.2$, 46.8, 48.6, 56.4, 56.7, 66.8, 108.6, 111.1, 120.0, 128.3, 128.4, 128.8, 132.3, 137.3, 148.2, 151.6, 156.1, 166.0. Anal. Calcd. for $\mathrm{C}_{21} \mathrm{H}_{24} \mathrm{~N}_{2} \mathrm{O}_{4} \mathrm{C}, 68.46 ; \mathrm{H}, 6.57 ; \mathrm{N}, 7.60$; Found C, 68.22; H, 6.41; $\mathrm{N}, 7.47 \%$.

$\left(1 S^{*}, 1^{\prime} S^{*}\right)-1-\left[1^{\prime}\right.$-(Benzyloxycarbonylamino)ethyl]-6,7-dimethoxy-1,2,3,4-tetrahydroisoquinoline (8a)

To a stirred and ice-cooled solution of dihydroisoquinoline 7 (10.00 g, $27.1 \mathrm{mmol})$ in $\mathrm{MeOH}$ (200 mL), $\mathrm{NaBH}_{4}(3.10 \mathrm{~g}, 82 \mathrm{mmol})$ was added in small portions. The resulting mixture was stirred for $3 \mathrm{~h}$ with ice-water bath cooling and for $3 \mathrm{~h}$ at ambient temperature, and then evaporated in vacuo. The residue was dissolved in $5 \% \mathrm{HCl}(150 \mathrm{~mL})$, and the solution was made alkaline with $20 \% \mathrm{NaOH}$ while cooled, and then extracted with $\mathrm{CHCl}_{3}(4 \times 150 \mathrm{~mL})$. The combined organic extracts were dried $\left(\mathrm{Na}_{2} \mathrm{SO}_{4}\right)$ and evaporated in vacuo to give an oily product, containing diastereomers $8 \mathbf{a}$ and $\mathbf{8 b}$ in a $c a .8: 1$ ratio. The oil crystallized on treatment with a 2:1 mixture of $n$-hexane and $\mathrm{Et}_{2} \mathrm{O}$. The crystalline product, which was filtered off and washed with $n$-hexane, proved to be diastereomerically pure 8a. The crude crystalline product was used in the next step without further purification.

Yield: $7.42 \mathrm{~g}(74 \%)$. An analytical sample of $8 \mathbf{a}$ was recrystallized from $\mathrm{Et}_{2} \mathrm{O}$ to give a white crystalline substance, mp 68-70 ${ }^{\circ} \mathrm{C} .{ }^{1} \mathrm{H}$ NMR $\left(400 \mathrm{MHz}, \mathrm{CDCl}_{3}\right): \delta_{\mathrm{H}} 1.35(\mathrm{~d}, 3 \mathrm{H}, J=6.8 \mathrm{~Hz}$, $\left.\mathrm{CH}_{3}\right), 2.50-2.60\left(\mathrm{~m}, 1 \mathrm{H}, \mathrm{ArCH}_{2}\right), 2.72-2.83\left(\mathrm{~m}, 1 \mathrm{H}, \mathrm{ArCH}_{2}\right), 2.88-2.96\left(\mathrm{~m}, 1 \mathrm{H}, \mathrm{NCH}_{2}\right), 3.32-$ $3.55\left(\mathrm{~m}, 2 \mathrm{H}, \mathrm{NCH}_{2}, \mathrm{NCH}\right), 3.83\left(\mathrm{~s}, 3 \mathrm{H}, \mathrm{OCH}_{3}\right), 3.85$ (s, 3H, OCH $\mathrm{OH}_{3}, 4.02$ (br s, 1H, NCH), 4.97$5.05\left(\mathrm{~m}, 1 \mathrm{H}, \mathrm{OCH}_{2}\right), 5.54$ (br s, $\left.1 \mathrm{H}, \mathrm{NH}\right), 6.58\left(\mathrm{~s}, 1 \mathrm{H}, \mathrm{C}_{6} H_{2}\right), 6.68\left(\mathrm{~s}, 1 \mathrm{H}, \mathrm{C}_{6} H_{2}\right), 7.28-7.34(\mathrm{~m}$, $\left.5 \mathrm{H}, \mathrm{C}_{6} \mathrm{H}_{5}\right) .{ }^{13} \mathrm{C} \mathrm{NMR}\left(100 \mathrm{MHz}, \mathrm{CDCl}_{3}\right): \delta_{\mathrm{C}} 19.0,30.0,43.2,56.2,56.4,59.8,66.6,109.4$, 112.0, 127.0, 128.0, 128.2, 128.5, 128.9, 137.3, 147.8, 147.9, 156.5. Anal. Calcd. for $\mathrm{C}_{21} \mathrm{H}_{26} \mathrm{~N}_{2} \mathrm{O}_{4} \mathrm{C}, 68.09 ; \mathrm{H}, 7.07$; N, 7.56; Found C, 67.76; H, 6.88; N, 7.24\%.

$\left(1 S^{*}, 1^{\prime} S^{*}\right)-1-(1 '$-Aminoethyl)-6,7-dimethoxy-1,2,3,4-tetrahydroisoquinoline dihydrobromide (9.2HBr)

A mixture of compound 8a $(6.50 \mathrm{~g}, 17.5 \mathrm{mmol})$ and $33 \% \mathrm{HBr}$ in $\mathrm{AcOH}(20 \mathrm{~mL})$ was heated gently in a flask equipped with a $\mathrm{CaCl}_{2}$ tube, with occasional shaking, until all of the substance had dissolved. The bubbling solution was left to stand at ambient temperature for $30 \mathrm{~min}$, and $\mathrm{Et}_{2} \mathrm{O}(25 \mathrm{~mL})$ was then added. The yellow crystals of the dihydrobromide of 9 which formed were filtered off, washed with EtOAc and $\mathrm{Et}_{2} \mathrm{O}$, dried and recrystallized from 95\% $\mathrm{MeOH}_{-} \mathrm{Et}_{2} \mathrm{O}$. Beige crystalline substance, yield: $5.78 \mathrm{~g}(83 \%), \mathrm{mp} 268-271{ }^{\circ} \mathrm{C} .{ }^{1} \mathrm{H}$ NMR $\left(400 \mathrm{MHz}, \mathrm{D}_{2} \mathrm{O}\right): \delta_{\mathrm{H}}$ $1.46\left(3 \mathrm{H}, \mathrm{d}, J=7.0 \mathrm{~Hz}, \mathrm{CH}_{3}\right), 2.98-3.17\left(2 \mathrm{H}, \mathrm{m}, \mathrm{ArCH}_{2}\right), 3.37-3.48\left(1 \mathrm{H}, \mathrm{m}, \mathrm{NCH}_{2}\right), 3.63-3.73$ $\left(1 \mathrm{H}, \mathrm{m}, \mathrm{NCH}_{2}\right), 3.88\left(6 \mathrm{H}, \mathrm{s}, 2 \times \mathrm{OCH}_{3}\right), 4.25-4.36(1 \mathrm{H}, \mathrm{m}, \mathrm{NCH}), 4,91(1 \mathrm{H}, \mathrm{d}, J=4.9 \mathrm{~Hz}$, $\mathrm{NCH}), 6.94\left(1 \mathrm{H}, \mathrm{s}, \mathrm{C}_{6} \mathrm{H}_{2}\right), 6.98\left(1 \mathrm{H}, \mathrm{s}, \mathrm{C}_{6} \mathrm{H}_{2}\right) .{ }^{13} \mathrm{C} \mathrm{NMR}\left(100 \mathrm{MHz}, \mathrm{D}_{2} \mathrm{O}\right): \delta_{\mathrm{C}} 24.5,40.7,49.3$, 56.4, 56.6, 57.3, 110.4, 113.0, 118.9, 126.7, 148.3, 149.6. Anal. Calcd. for $\mathrm{C}_{13} \mathrm{H}_{22} \mathrm{Br}_{2} \mathrm{~N}_{2} \mathrm{O}_{2} \mathrm{C}$, 39.22; H, 5.57; N, 7.04; Found C, 38.87; H, 5.20; N, $6.98 \%$. 
Pure diamine base 9 was obtained from the above dihydrobromide by alkaline treatment (20\% $\mathrm{NaOH})$, extraction $\left(\mathrm{CH}_{2} \mathrm{Cl}_{2}\right)$ and evaporation under reduced pressure. The free base was dried in a vacuum desiccator for $24 \mathrm{~h}$ before further transformations.

General procedure for the preparation of tetra-, penta- and hexa-cyclic lactams 11-20 and 22

A mixture of diamine 9 or $\mathbf{1 0}$ or 21 ( $3 \mathrm{mmol})$ and the corresponding $\gamma$ - or $\delta$-oxo acid $(3 \mathrm{mmol})$ was refluxed in toluene $(40 \mathrm{~mL})$ until no more starting materials could be detected by TLC (3-12 h). In case of 21, some crystals of $p$-toluenesulfonic acids were also added to the reaction mixture prior to reflux. The solvent was then evaporated off and the oily or solid residue (the NMR spectrum of which was applied for determination of the diastereomeric ratios) was purified by means of column chromatography.

The ${ }^{1} \mathrm{H}$ NMR (11, 16-18: $400 \mathrm{MHz}$; 12-15, 19-20, 22: $\left.500 \mathrm{MHz}, \mathrm{CDCl}_{3}\right)$ and ${ }^{13} \mathrm{C} \mathrm{NMR}(100$ $\mathrm{MHz}, \mathrm{CDCl}_{3}$ ) data of compounds 11-20 and $\mathbf{2 2}$ are presented in Tables 1-3.

8,9-Dimethoxy-3a,11-dimethyl-3,3a,5,6,10b,11-hexahydropyrrolo[2',1':2,3]imidazo[5,1-a]isoquinolin-1(2H)-one (11). Eluent: EtOAc : $\mathrm{MeOH}=4: 1$, yield: $0.73 \mathrm{~g}(77 \%)$, white crystalline substance, $\mathrm{mp} 192-193{ }^{\circ} \mathrm{C}$ (EtOAc). MS/MS from $[\mathrm{M}+\mathrm{H}]^{+}(33.57-41.03 \mathrm{eV}): 220$ (9), 205 (20), 204 (30), 202 (13), 193 (16), 192 (98), 190 (62), 189 (20), 177 (55), 176 ( (94), 174 (100), 162 (20), 160 (26), 159 (25), 148 (68), 147 (21), 146 (18), 145 (19), 133 (10), 131 (48), 130 (10), 129 (10), 126 (12), 117 (12), 115 (9), 105 (7), 98 (14). Anal. Calcd. for $\mathrm{C}_{18} \mathrm{H}_{24} \mathrm{~N}_{2} \mathrm{O}_{3} \mathrm{C}$, $68.33 ; \mathrm{H}, 7.65 ; \mathrm{N}, 8.85$; Found C, 68.09; H, 7.48; N, 8.72\%.

8,9-Dimethoxy-11-methyl-3a-phenyl-3,3a,5,6,10b,11-hexahydropyrrolo[2',1':2,3]imidazo[5,1-a]isoquinolin-1(2H)-one (12). Eluent: EtOAc, yield: $0.77 \mathrm{~g}(68 \%)$, beige solid, mp 155-158 ${ }^{\circ} \mathrm{C}$. Anal. Calcd. for $\mathrm{C}_{23} \mathrm{H}_{26} \mathrm{~N}_{2} \mathrm{O}_{3} \mathrm{C}, 72.99 ; \mathrm{H}, 6.92 ; \mathrm{N}, 7.40$; Found C, 72.82; H, 6.75; N, 7.27\%.

8,9-Dimethoxy-3a,11-dimethyl-3,3a,5,6,11,12-hexahydro-10bH-pyrrolo[2',1':2,3]pyrimido[6,1-a]isoquinolin-1(2H)-one (13): Eluent: EtOAc, yield: $0.58 \mathrm{~g}$ (59\%), beige crystalline substance, mp 139-141 ${ }^{\circ} \mathrm{C}$. Anal. Calcd. for $\mathrm{C}_{19} \mathrm{H}_{26} \mathrm{~N}_{2} \mathrm{O}_{3} \mathrm{C}, 69.06 ; \mathrm{H}, 7.93 ; \mathrm{N}, 8.48$; Found C, $68.85 ; \mathrm{H}, 7.62 ; \mathrm{N}, 8.30 \%$.

8,9-Dimethoxy-11-methyl-3a-phenyl-3,3a,5,6,11,12-hexahydro-10bH-pyrrolo[2', $\left.1^{\prime}: 2,3\right]$ pyrimido[6,1-a]isoquinolin-1(2H)-one (14). Eluent: EtOAc, yield: $0.76 \mathrm{~g}$ (65\%), white crystalline substance, mp 233-234 ${ }^{\circ} \mathrm{C}\left(i \mathrm{Pr}_{2} \mathrm{O}-\mathrm{EtoAc}\right)$. Anal. Calcd. for $\mathrm{C}_{24} \mathrm{H}_{28} \mathrm{~N}_{2} \mathrm{O}_{3} \mathrm{C}, 73.44 ; \mathrm{H}, 7.19 ; \mathrm{N}$, 7.14; Found C, 73.19; H, 7.03; N, 6.98\%.

9,10-Dimethoxy-12-methyl-4a-phenyl-2,3,4,4a,6,7,11b,12-octahydro-1H-pyrido[2',1':2,3]imidazo[5,1-a]isoquinolin-1-one (15). Eluent: EtOAc, yield: $0.74 \mathrm{~g}$ (63\%), beige solid, mp 174$177{ }^{\circ} \mathrm{C}$. Anal. Calcd. for $\mathrm{C}_{24} \mathrm{H}_{28} \mathrm{~N}_{2} \mathrm{O}_{3} \mathrm{C}, 73.44 ; \mathrm{H}, 7.19 ; \mathrm{N}, 7.14$; Found $\mathrm{C}, 73.61 ; \mathrm{H}, 7.06$; N, $7.12 \%$.

9,10-Dimethoxy-12-methyl-4a-phenyl-2,3,4,4a,6,7,12,13-octahydro-1H,11b $H$-pyrido[2',1':2,3]pyrimido[6,1-a]isoquinolin-1-one (16). Eluent: EtOAc, yield: $0.63 \mathrm{~g}$ (52\%), white crystalline substance, mp 202-204 ${ }^{\circ} \mathrm{C}$. Anal. Calcd. for $\mathrm{C}_{25} \mathrm{H}_{30} \mathrm{~N}_{2} \mathrm{O}_{3} \mathrm{C}, 73.86 ; \mathrm{H}, 7.44 ; \mathrm{N}, 6.89$; Found C, 73.61; H, 7.38; N, 6.62\%. 
2,3-Dimethoxy-5-methyl-4b,5,13,14-tetrahydroisoindolo[1',2' :2,3]imidazo[5,1-a]isoquinolin-7(11bH)-one (17). Eluent: EtOAc, yield: $0.78 \mathrm{~g}(74 \%)$, white crystalline substance, mp 186-187 ${ }^{\circ} \mathrm{C}$. Anal. Calcd. for $\mathrm{C}_{21} \mathrm{H}_{22} \mathrm{~N}_{2} \mathrm{O}_{3} \mathrm{C}, 71.98 ; \mathrm{H}, 6.33 ; \mathrm{N}, 7.99$; Found C, 72.15; H, 6.10; $\mathrm{N}, 7.82 \%$.

\section{2,3-Dimethoxy-5-methyl-11b-phenyl-4b,5,13,14-tetrahydroisoindolo[1',2':2,3]imidazo-} [5,1-a] isoquinolin-7(11bH)-one (18). Eluent: EtOAc, yield: $0.81 \mathrm{~g}$ (63\%), white crystalline substance, mp 174-175 ${ }^{\circ} \mathrm{C}\left(\mathrm{Et}_{2} \mathrm{O}\right)$. Anal. Calcd. for $\mathrm{C}_{27} \mathrm{H}_{26} \mathrm{~N}_{2} \mathrm{O}_{3} \mathrm{C}, 76.03 ; \mathrm{H}, 6.14 ; \mathrm{N}, 6.57$; Found C, 76.26; H, 6.02; N, 6.49\%.

2,3-Dimethoxy-5-methyl-5,6,14,15-tetrahydro-4bH-isoindolo $\left[1^{\prime}, 2\right.$ ' :2,3]pyrimido[6,1-a $]$ isoquinolin-8(12bH)-one (19). Eluent: EtOAc, yield: $0.83 \mathrm{~g}(76 \%)$, white crystalline substance, $\mathrm{mp}$ 209-210 ${ }^{\circ} \mathrm{C}\left(i \mathrm{Pr}_{2} \mathrm{O}-\mathrm{EtOAc}\right)$. Anal. Calcd. for $\mathrm{C}_{22} \mathrm{H}_{24} \mathrm{~N}_{2} \mathrm{O}_{3} \mathrm{C}, 72.50 ; \mathrm{H}, 6.64 ; \mathrm{N}, 7.69$; Found C, 72.26; H, 6.41; N, 7.74\%.

2,3-Dimethoxy-5-methyl-12b-phenyl-5,6,14,15-tetrahydro-4bH-isoindolo[1',2':2,3]pyrimido[6,1-a] isoquinolin-8(12bH)-one (20). Eluent: EtOAc, yield: $0.77 \mathrm{~g}(58 \%)$, beige solid, mp 225-227 ${ }^{\circ} \mathrm{C}\left(i \mathrm{Pr}_{2} \mathrm{O}-\mathrm{EtOAc}\right)$. Anal. Calcd. for $\mathrm{C}_{28} \mathrm{H}_{28} \mathrm{~N}_{2} \mathrm{O}_{3} \mathrm{C}, 76.34 ; \mathrm{H}, 6.41 ; \mathrm{N}, 3,6.36$; Found C, 76.10; H, 6.29; N, 6.14\%.

2,3-Dimethoxy-16,17-dihydro-4bH-isoindolo[2,1-a]isoquinolino[2,1-c]quinazolin-10(14bH)one (22). Eluent: EtOAc : $\mathrm{MeOH} 4$ : 1, yield: $0.72 \mathrm{~g} \mathrm{(60 \% ),} \mathrm{white} \mathrm{crystalline} \mathrm{substance,} \mathrm{mp} \mathrm{245-}$ $246{ }^{\circ} \mathrm{C}$ (EtOAc-EtOH). Anal. Calcd. for $\mathrm{C}_{25} \mathrm{H}_{22} \mathrm{~N}_{2} \mathrm{O}_{3} \mathrm{C}, 75.36 ; \mathrm{H}, 5.57 ; \mathrm{N}, 7.03$; Found C, 75.42; H, 5.39; N, 6.84\%.

\section{Acknowledgements}

ZZ and LL thank TÁMOP-4.2.1/B-09/1/KONV-2010-0005 for financial support.

\section{References and Notes}

1. (a) Meyers, A. I.; Brengel, G. P. Chem. Commun. 1997, 1. (b) Csende, F.; Stájer, G. Heterocycles 2000, 53, 1379. (c) Csende, F.; Stájer, G. Curr. Org. Chem. 2005, 9, 1261.

2. (a) Tietze, L. F. Chem. Rev. 1996, 96, 115. (b) Tietze, L. F.; Rackelmann, N. Pure Appl. Chem. 2004, 76, 1967. (c) Padwa, A.; Bur, S. K. Tetrahedron 2007, 63, 5341. (d) Alba, A.N.; Companyo, X.; Viciano, M.; Rios, R. Curr. Org. Chem. 2009, 13, 1432.

3. (a) Meyers, A. I.; Downing, S. V.; Weiser, M. J. J. Org. Chem. 2001, 66, 1413. (b) Lázár, L.; Fülöp, F. Eur. J. Org. Chem. 2003, 3025.

4. (a) Groaning, M. D.; Meyers, A. I. Tetrahedron 2000, 56, 9843. (b) Escolano, C.; Amat, M.; Bosch J. Chem. Eur. J. 2006, 12, 8198. (c) Amat, M.; Perez, M.; Bosch, J. Synlett 2011, 143. (d) Amat, M.; Arróniz, C.; Molins, E.; Escolano, C.; Bosch, J. Org. Biomol. Chem. 2011, 9, 2175. 
5. (a) Lázár, L; Kivelä, H.; Pihlaja, K.; Fülöp, F. Tetrahedron Lett. 2004, 45, 6199. (b) Kivelä, H.; Tähtinen, P.; Martiskainen, O.; Pihlaja, K.; Lázár, L.; Vigóczki, E.; Fülöp, F. J. Mol. Struct. 2010, 983, 62.

6. (a) Zalán, Z.; Martinek, T. A.; Lázár, L.; Sillanpää, R., Fülöp, F. Tetrahedron 2006, 62, 2883. (b) Schuster, I.; Koch, A.; Heydenreich, M.; Kleinpeter, E.; Forró, E.; Lázár, L.; Sillanpää, R.; Fülöp, F. Eur. J. Org. Chem. 2008, 1464. (c) Kleinpeter, E., Szatmári, I.; Lázár, L., Koch, A.; Heydenreich, M.; Fülöp, F. Tetrahedron 2009, 65, 8021. (d) Vigóczki, E., Hetényi, A., Lázár, L.; Fülöp, F. ARKIVOC 2009, (xii), 8.

7. Zalán, Z.; Martinek, T. A.; Lázár, L.; Fülöp, F. Tetrahedron 2003, 59, 9117.

8. von Nussbaum, F.; Miller, B.; Wild, S.; Hilger, C. S.; Schumann, S.; Zorbas, H.; Beck, W.; Steglich, W. J. Med. Chem. 1999, 42, 3478.

9. All of the prepared compounds are racemic. For a better stereochemical comparison of the polycyclic lactams, diamine 9 was depicted on the Schemes as $\left(1 S^{*}, 1^{\prime} S^{*}\right)$, while $\mathbf{1 0}$ as $\left(1 R^{*}, 1^{\prime} R^{*}\right)$ diastereomer. 